\title{
Enabling constrained spherical deconvolution and diffusional variance decomposition with tensor-valued diffusion MRI
}

\author{
Philippe Karan $^{\mathrm{a}, *}$, Alexis Reymbaut ${ }^{\mathrm{a}}$, Guillaume Gilbert ${ }^{\mathrm{b}}$, Maxime Descoteaux ${ }^{\mathrm{a}}$ \\ ${ }^{a}$ Sherbrooke Connectivity Imaging Laboratory (SCIL), Université de Sherbrooke, Canada \\ ${ }^{b}$ MR Clinical Science, Philips Healthcare Canada, Markham, ON L6C 2S3, Canada
}

\begin{abstract}
Diffusion tensor imaging (DTI) is widely used to extract valuable tissue measurements and white matter (WM) fiber orientations, even though its lack of specificity is now well-known, especially for WM fiber crossings. Models such as constrained spherical deconvolution (CSD) take advantage of high angular resolution diffusion imaging (HARDI) data to compute fiber orientation distribution functions (fODF) and tackle the orientational part of the DTI limitations. Furthermore, the recent introduction of tensor-valued diffusion MRI allows for diffusional variance decomposition (DIVIDE), opening the door to the computation of measures more specific to microstructure than DTI measures, such as microscopic fractional anisotropy $(\mu \mathrm{FA})$. However, tensor-valued diffusion MRI data is not mathematically compatible with latest versions of CSD and the impacts of such atypical data on fODF reconstruction with CSD are yet to be studied. In this work, we lay down the mathematical and computational foundations of a tensor-valued CSD and use simulated data to explore the effects of various combinations of diffusion encodings on the angular resolution of extracted fOFDs. We also compare the combinations with regards to their performance at producing accurate and precise $\mu \mathrm{FA}$ with DIVIDE, and present an optimised protocol for both methods. We show that our proposed protocol enables the reconstruction of both fODFs and $\mu \mathrm{FA}$ on in vivo data.
\end{abstract}

Keywords: Diffusion MRI, Tensor-valued dMRI, White Matter Fiber Crossing, Constrained Spherical Deconvolution, Diffusional Variance Decomposition

\section{Introduction}

Diffusion MRI (dMRI) [Le Bihan and Breton, 1985] allows for non-invasive probing of the diffusion of water molecules in tissues such as the human brain. In particular, diffusion tensor imaging (DTI) [Basser et al., 1994] models the average voxel content with a diffusion tensor to get access to valuable information about the intra-voxel diffusion profile. Indeed, the diffusion tensor gives insight into the orientation of the white matter (WM) fibers and leads to the calculation of DTI measures, such as the well known mean

*2500, boul. de l'Université, Sherbrooke (Québec) Canada, J1K 2R1

Email address: Philippe.Karan@USherbrooke.ca (Philippe Karan) 
diffusivity (MD), fractional anisotropy (FA) [Basser and Pierpaoli, 1996], axial diffusivity $(\mathrm{AD})$ and radial diffusivity $(\mathrm{RD})$. While the orientation of the diffusion tensor and the computation of these measures are great tools for the study of WM in the brain and are widely used, important limitations were pointed out [Wheeler-Kingshott and Cercignani, 2009; Jones and Cercignani, 2010]. Furthermore, Tuch et al. [2002] showed that DTI cannot properly model a voxel containing multiple WM fiber orientations. This leads to the FA drop in WM fiber crossings and to a counter-intuitive FA increase in, e.g., Alzheimer lesions [Douaud et al., 2011; Teipel et al., 2014], resulting in a very ambiguous interpretation of this measure in what is estimated to represent 60 to $90 \%$ of voxels in a typical whole-brain scan [Descoteaux, 2008; Jeurissen et al., 2013; Volz et al., 2018]. To obtain a better WM fiber orientation profile, Tuch et al. [2002] proposed the high angular resolution diffusion imaging (HARDI) idea, which gave birth to many HARDIbased methods such as constrained spherical deconvolution (CSD) [Tournier et al., 2004, 2007; Descoteaux et al., 2009], used to extract a fiber orientation distribution function (fODF) from HARDI data. These fODFs can then serve as guides for tractography algorithms [Mori et al., 1999; Poupon et al., 2000; Catani et al., 2002], allowing structural connectivity human brain mapping studies and applications.

An extension of DTI, diffusion kurtosis imaging (DKI) [Jensen et al., 2005], enables to estimate a first measure of tissue heterogeneity: the mean kurtosis. However, this mean kurtosis is not specific, as it originates from both microscopic anisotropy (probing pure cell elongation) and isotropic heterogeneity (variance of isotropic diffusivities or variance of cell densities). A new dMRI technique introduced in the mid-2010s, called tensor-valued dMRI [Eriksson et al., 2013, 2015; Westin et al., 2014, 2016] or b-tensor encoding, shows great promise in alleviating the lack of specificity of conventional dMRI techniques such as DTI or DKI. In particular, Lasič et al. [2014] proposed a way to disentangle the isotropic and anisotropic components of the diffusional variance, leading to new measures of microscopic anisotropy and isotropic heterogeneity. Recent papers [Nilsson et al., 2020; Naranjo et al., 2021] have even established clinically feasible tensor-valued dMRI scans providing sufficient data to compute these new measures with similar methods. Moreover, many studies have investigated the potential of tensor-valued diffusion encoding for microstructural characterizations of brain tumors and neurodegenerative diseases [Szczepankiewicz et al., 2015, 2016; Andersen et al., 2020; Kamiya et al., 2020; Nilsson et al., 2020].

Several studies have accounted for sub-voxel WM fascicle orientations while employing tensor-valued diffusion encoding to capture the aforementioned diffusion measures [Cottaar et al., 2020; Reymbaut et al., 2020a,b, 2021; de Almeida Martins et al., 2021]. However, much remains to be done in evaluating the effects of various diffusion encodings on fODF reconstruction with CSD. Indeed, the standard single-shell single-tissue CSD (ssst-CSD) [Tournier et al., 2007; Descoteaux et al., 2009] does not allow for the use of tensor-valued dMRI data and the current state of the literature does not provide any CSD model that can use such data as input, except for one conference abstract [Jeurissen and Szczepankiewicz, June 2018]. Nevertheless, the work of Jeurissen et al. [2014] on extending the ssst-CSD model to a multi-shell multi-tissue CSD (msmt-CSD) model is a great example of the flexibility of CSD, allowing it to take multi-shell dMRI data as input and accurately differentiate brain tissues.

This paper establishes the foundations of a tensor-valued diffusion encoding CSD model, as a mathematical extension of msmt-CSD, enabling the reconstruction of fODFs 
using tensor-valued dMRI data. The impacts of different combinations of b-tensor shapes, namely linear, planar and spherical tensors, on the reconstruction of fODFs are explored using the adapted CSD model on simulated data. In parallel, these combinations are also challenged with a method for disentangling the diffusional variance, and performances on both methods are compared to lead to the overall best combination. It is important to propose a technique that can extract both accurate crossing fibers as well as advanced microstructural maps from tensor-valued dMRI. Thus, we propose a 10 minutes long tensor-valued dMRI protocol enabling an accurate reconstruction of the fODFs while also allowing the computation of the b-tensor encoding microstructure measures. The performances of this final protocol are shown on in vivo data as a demonstration of the new tensor-valued diffusion encoding CSD model's potential.

\section{Theory}

Assuming a non diffusion-weighted (DW) signal $S_{0}$, the DW signal $S_{i}$ for an acquisition direction $i$ is defined as

$$
S_{i}=S_{0} \int_{\mathbf{D}} P(\mathbf{D}) e^{-\mathbf{B}_{i}: \mathbf{D}} \mathrm{d} \mathbf{D}
$$

where $P(\mathbf{D})$ is the intra-voxel diffusion tensor distribution (DTD) and $\mathbf{B}_{i}: \mathbf{D}$ denotes the Frobenius inner product between the so-called b-tensor $\mathbf{B}_{i}$ and a diffusion tensor $\mathbf{D}$ :

$$
\mathbf{B}_{i}: \mathbf{D}=\sum_{m, n}\left[\mathbf{B}_{i}\right]_{m n}[\mathbf{D}]_{m n} .
$$

Note that this definition of the signal assumes a heterogeneous intra-voxel content, described by the DTD [Basser and Pajevic, 2003; Jian et al., 2007; Reymbaut, 2020].

\subsection{Diffusional variance from the DTD}

Following the formalism of Szczepankiewicz [2016], different measures can be calculated from the DTD $P(\mathbf{D})$. For instance, the average (denoted by $\langle\cdot\rangle$ ) of the diffusion tensors across the DTD in a voxel gives a voxel-scale diffusion tensor $\langle\mathbf{D}\rangle$, the same one that is at the center of the DTI model. From this, it is well known that the mean diffusivity $(\mathrm{MD})$ is obtained according to $\mathrm{MD}=\mathrm{E}_{\lambda}[\langle\mathbf{D}\rangle]$, where $\mathrm{E}_{\lambda}[\cdot]$ is the average over tensor eigenvalues $\lambda$. However, the MD can also be calculated as the average of all isotropic diffusivities $\mathrm{E}_{\lambda}[\mathbf{D}]$ in the DTD:

$$
\mathrm{MD}=\left\langle\mathrm{E}_{\lambda}[\mathbf{D}]\right\rangle
$$

The DTD also contains information about the isotropic variance $V_{\mathrm{I}}$, reflecting the isotropic heterogeneity of the voxel, and the anisotropic variance $V_{\mathrm{A}}$, describing the microscopic anisotropy of the voxel. These diffusional variances are calculated as follow, with $\mathrm{V}_{\lambda}[\cdot]$ being the population variance of tensor eigenvalues $\lambda$ :

$$
\begin{gathered}
V_{\mathrm{I}}=\left\langle\mathrm{E}_{\lambda}[\mathbf{D}]^{2}\right\rangle-\mathrm{E}_{\lambda}[\langle\mathbf{D}\rangle]^{2}, \\
V_{\mathrm{A}}=\frac{2}{5}\left\langle\mathrm{~V}_{\lambda}[\mathbf{D}]\right\rangle . \\
3
\end{gathered}
$$


Diffusional variance decomposition (DIVIDE) can be used to extract $\mathrm{MD}, V_{\mathrm{I}}$ and $V_{\mathrm{A}}$ from tensor-valued dMRI data with at least two different b-tensor encodings Lasič et al. [2014]; Szczepankiewicz et al. [2015, 2016]. This method estimates the isotropic and anisotropic variances of the DTD by fitting the following inverse Laplace transform of the gamma distribution function [Röding et al., 2012] to powder-averaged tensor-valued dMRI data $\bar{S}_{i}$ :

$$
\bar{S}_{i}=S_{0}\left(1+b_{i} \frac{V_{\mathrm{D}}}{\mathrm{MD}}\right)^{-\frac{\mathrm{MD}^{2}}{V_{\mathrm{D}}}}
$$

where $V_{\mathrm{D}}=V_{\mathrm{I}}+b_{\Delta, i}^{2} V_{\mathrm{A}}$ is the diffusional variance and $b_{\Delta, i}$ is a factor describing the encoding shape $\left(b_{\Delta, i}=1\right.$ for linear, $b_{\Delta, i}=-0.5$ for planar and $b_{\Delta, i}=0$ for spherical). This disentanglement of the diffusional variances enables the definition of the microscopic fractional anisotropy $\mu \mathrm{FA}$ [Lasič et al., 2014; Reymbaut, 2020] as:

$$
\mu \mathrm{FA}=\sqrt{\frac{3}{2} \cdot \frac{\left\langle V_{\lambda}[\mathbf{D}]\right\rangle}{\left\langle\mathrm{E}_{\lambda}[\mathbf{D}]^{2}\right\rangle+\left\langle V_{\lambda}[\mathbf{D}]\right\rangle}}=\sqrt{\frac{3}{2} \cdot \frac{\frac{5}{2} V_{\mathrm{A}}}{V_{\mathrm{I}}+\mathrm{MD}^{2}+\frac{5}{2} V_{\mathrm{A}}}} .
$$

This new measure is thus representative of the average anisotropy computed across all microscopic environments in a voxel, with values going from 0 (purely isotropic cellular structures) to 1 (purely stick-like cellular structures). In comparison, the typical FA computed from DTI gives information about the anisotropy observed at the voxel scale, consequently depending on the orientation coherence of the underlying cellular structures, which is quantified by the order parameter (OP) [Lasič et al., 2014]. The $\mu$ FA becomes equal to the FA in voxels where all microscopic environments are perfectly identical and ordered.

\subsection{Tensor-valued constrained spherical deconvolution}

In the case of a single homogeneous axisymmetric fiber with orientation $\mathbf{u}_{k} \equiv\left(\theta_{k}, \phi_{k}\right)$, the DTD from equation 1 becomes a Dirac distribution peaked at a single diffusion tensor $\mathbf{D}_{k}$ of main eigenvector $\mathbf{u}_{k}$, axial and radial diffusivities $D_{\|}$and $D_{\perp}$. The Frobenius inner product from equation 2 then becomes

$$
\mathbf{B}_{i}: \mathbf{D}_{k}=\frac{b_{i, S}}{3}\left(D_{\|}+2 D_{\perp}\right)+b_{i, L}\left(D_{\|} \cos ^{2} \beta_{i k}+D_{\perp} \sin ^{2} \beta_{i k}\right),
$$

where the angle $\beta_{i k}$ separating the orientation of the b-tensor $\mathbf{e}_{i} \equiv\left(\Theta_{i}, \Phi_{i}\right)$ and the fiber orientation $\mathbf{u}_{k}$ is given by the spherical law of cosines

$$
\cos \beta_{i k}=\cos \Theta_{i} \cos \theta_{k}+\sin \Theta_{i} \sin \theta_{k} \cos \left(\Phi_{i}-\phi_{k}\right) .
$$

The couple $\left(b_{i, S}, b_{i, L}\right)$ determines the b-tensor's encoding type such as

$$
\begin{cases}\left(b_{i, S}, b_{i, L}\right)=\left(0, b_{i}\right) & \text { for linear tensor encoding (LTE) } \\ \left(b_{i, S}, b_{i, L}\right)=\left(b_{i}, 0\right) & \text { for spherical tensor encoding (STE) } \\ \left(b_{i, S}, b_{i, L}\right)=\left(3 b_{i} / 2,-b_{i} / 2\right) & \text { for planar tensor encoding (PTE) }\end{cases}
$$

where $b_{i}$ is the typical b-value, given by $b_{i}=\operatorname{Tr}\left(\mathbf{B}_{i}\right)$, the trace of the b-tensor. 
With the DTD being a Dirac distribution peaked at a single diffusion tensor and substituting the Frobenius inner product from equation 8, equation 1 simplifies to

$$
S_{i}=S_{0} e^{-\frac{b_{i, S}}{3}\left(D_{\|}+2 D_{\perp}\right)-b_{i, L}\left(D_{\|} \cos ^{2} \beta_{i k}+D_{\perp} \sin ^{2} \beta_{i k}\right)} \equiv K_{\epsilon_{i}}\left(b_{i} ; \mathbf{e}_{i}, \mathbf{u}_{k}\right),
$$

where we introduce the single fiber response function (FRF) or convolution kernel $K_{\epsilon_{i}}$, which changes according to the diffusion encoding of acquisition $i$, such that $\epsilon_{i} \in\{$ linear, planar, spherical $\}$. If multiple identical homogeneous axisymmetric fibers are present, the equation becomes an integral over the unit sphere $\mathbb{S}^{2}$

$$
S_{i}=\int_{\mathbb{S}^{2}} K_{\epsilon_{i}}\left(b_{i} ; \mathbf{e}_{i}, \mathbf{u}\right) f(\mathbf{u}) \mathrm{d}^{2} \mathbf{u},
$$

where the diffusion signal is modeled as the convolution of an fODF $f(\mathbf{u})$ with a fiber response function $K_{\epsilon_{i}}\left(b_{i} ; \mathbf{e}_{i}, \mathbf{u}\right)$. The fODF weighs different unit orientations of fiber in the signal, while the response function corresponds to the DW signal of a single fiber with orientation $\mathbf{u}$.

Using equation 10, the fiber response functions for linear, planar and spherical tensor encoding are

$$
\left\{\begin{array}{l}
K_{\text {linear }}\left(b_{i} ; \mathbf{e}_{i}, \mathbf{u}_{k}\right)=S_{0} \cdot \exp \left[-b_{i}\left(D_{\|} \cos ^{2} \beta_{i k}+D_{\perp} \sin ^{2} \beta_{i k}\right)\right] \\
K_{\text {planar }}\left(b_{i} ; \mathbf{e}_{i}, \mathbf{u}_{k}\right)=S_{0} \cdot \exp \left[-\frac{b_{i}}{2}\left(D_{\|} \sin ^{2} \beta_{i k}+D_{\perp}\left[1+\cos ^{2} \beta_{i k}\right]\right)\right] \\
K_{\text {spherical }}\left(b_{i} ; \mathbf{e}_{i}, \mathbf{u}_{k}\right)=S_{0} \cdot \exp \left[-\frac{b_{i}}{3}\left(D_{\|}+2 D_{\perp}\right)\right]
\end{array}\right.
$$

Figure 1 shows these encoding dependent theoretical FRFs for multiple b-values and tissues, namely white matter, grey matter (GM) and the cerebrospinal fluid (CSF), from diffusivity values taken from the literature and real data examples (see section 3.4).

The fODF is usually expressed as a linear combination of $N_{\mathrm{SH}}$ basis functions $Y_{j}(\mathbf{u})$, such as spherical harmonics $(\mathrm{SH})$, leading to

$$
f(\mathbf{u})=\sum_{j=1}^{N_{\mathrm{SH}}} w_{j} Y_{j}(\mathbf{u}),
$$

where $w_{j}$ are the $\mathrm{SH}$ coefficients in the case of an $\mathrm{SH}$ basis. Applying this to equation 12 then gives

$$
S_{i}=\sum_{j=1}^{N_{\mathrm{SH}}} w_{j} \int_{\mathbb{S}^{2}} K_{\epsilon_{i}}\left(b_{i} ; \mathbf{e}_{i}, \mathbf{u}\right) Y_{j}(\mathbf{u}) \mathrm{d}^{2} \mathbf{u},
$$

which can be written simply as a linear problem:

$$
\mathbf{S}=\mathbf{K} \cdot \mathbf{w}
$$

where the following matrix $\mathbf{K}$ and column vectors $\mathbf{S}$ and $\mathbf{w}$ read:

$$
[\mathbf{S}]_{i}=S_{i} \quad[\mathbf{K}]_{i j}=\int_{\mathbb{S}^{2}} K_{\epsilon_{i}}\left(b_{i} ; \mathbf{e}_{i}, \mathbf{u}\right) Y_{j}(\mathbf{u}) \mathrm{d}^{2} \mathbf{u} \quad[\mathbf{w}]_{j}=w_{j}
$$

Note that in the case of a linear encoding only acquisition, equation 15 falls back to the classic single-shell or multi-shell formulation, with a single linear fiber response function 
bioRxiv preprint doi: https://doi.org/10.1101/2021.04.07.438845; this version posted May 25, 2021. The copyright holder for this preprint (which was not certified by peer review) is the author/funder, who has granted bioRxiv a license to display the preprint in perpetuity. It is made available under aCC-BY 4.0 International license.

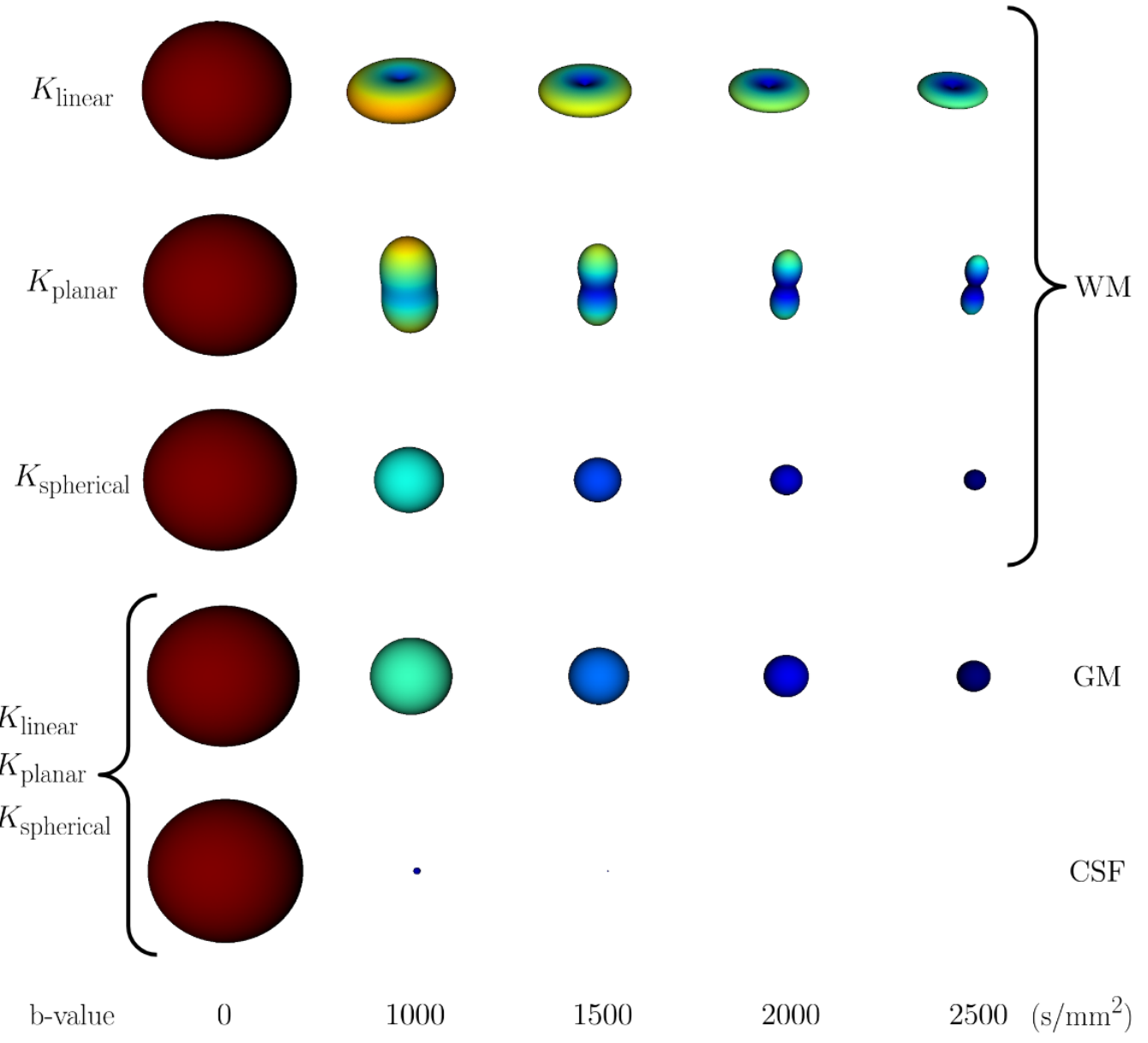

Figure 1: Visualisation of fiber response functions (FRF) $K_{\epsilon_{i}}$ computed from equation 13 for different b-values and tissues. The WM tissue, defined by $D_{\|}=1.7 \times 10^{-3} \mathrm{~mm}^{2} / \mathrm{s}$ and $D_{\perp}=0.3 \times 10^{-3} \mathrm{~mm}^{2} / \mathrm{s}$, shows varying FRFs for LTE, PTE and STE. For GM $\left(D_{\|}=D_{\perp}=0.6 \times 10^{-3} \mathrm{~mm}^{2} / \mathrm{s}\right)$ and CSF $\left(D_{\|}=D_{\perp}=3.0 \times 10^{-3} \mathrm{~mm}^{2} / \mathrm{s}\right)$, all tensor encodings produce the same FRF. Every fiber response function is calculated using $S_{0}=1$ and a diffusion tensor pointing in the $z$-axis. The FRFs for CSF are too small to be visible at $b \geq 2000 \mathrm{~s} / \mathrm{mm}^{2}$, because the diffusivities are high and lead to the amplitude of each $b>1000 \mathrm{~s} / \mathrm{mm}^{2}$ losing a factor of approximately 4.5 from the amplitude of the previous shell.

per b-value. The linearized problem of equation 16 can then be solved using the msmtCSD model developed by Jeurissen et al. [2014]. In this paper, the authors expand the ssst-CSD method from Tournier et al. [2007]; Descoteaux et al. [2009] to allow $m$ shells 
and $n$ tissues, resulting in the following constrained linear least squares problem:

$$
\begin{gathered}
\left(\begin{array}{c}
\hat{\mathbf{w}}_{1} \\
\vdots \\
\hat{\mathbf{w}}_{n}
\end{array}\right)=\arg \underset{\min }{\left(\begin{array}{c}
\mathbf{w}_{1} \\
\vdots \\
\mathbf{w}_{n}
\end{array}\right)}\left[\frac{1}{2}\left\|\left(\begin{array}{ccc}
\mathbf{K}_{1,1} & \ldots & \mathbf{K}_{1, n} \\
\vdots & \ddots & \vdots \\
\mathbf{K}_{m, 1} & \ldots & \mathbf{K}_{m, n}
\end{array}\right) \cdot\left(\begin{array}{c}
\mathbf{w}_{1} \\
\vdots \\
\mathbf{w}_{n}
\end{array}\right)-\left(\begin{array}{c}
\mathbf{S}_{1} \\
\vdots \\
\mathbf{S}_{m}
\end{array}\right)\right\|_{2}\right] \\
\text { fulfilling the condition } \quad\left(\begin{array}{ccc}
\mathbf{A}_{1} & 0 & 0 \\
0 & \ddots & 0 \\
0 & 0 & \mathbf{A}_{n}
\end{array}\right) \cdot\left(\begin{array}{c}
\mathbf{w}_{1} \\
\vdots \\
\mathbf{w}_{n}
\end{array}\right) \geq \mathbf{0},
\end{gathered}
$$

where $\mathbf{A}_{j}$ is the matrix relating the coefficients $\mathbf{w}_{j}$ to the fODF amplitudes. Solving this equation leads to the unknown vectors of coefficients of the fODF, with one vector for each tissue. In the case of $m=n=1$, this equation goes back to the ssst-CSD formalism.

\section{Methods}

\subsection{Implementation of tensor-valued constrained spherical deconvolution}

In the case of tensor-valued dMRI data, the linearized problem of equation 16 can be solved using an extension of equation 18 that enables CSD with multiple tensor-valued encodings, a method we call multi-encoding msmt-CSD (memsmt-CSD). This allows for $m_{\epsilon}$ shells per encoding $\epsilon$, and $n$ tissues. The convolution kernels then become a concatenation of the different encoding kernels, such as the linear ( $\mathbf{K}^{\mathrm{L}}$ with $m_{\mathrm{L}}$ shells), planar $\left(\mathbf{K}^{\mathrm{P}}\right.$ with $m_{\mathrm{P}}$ shells) and spherical $\left(\mathbf{K}^{\mathrm{S}}\right.$ with $m_{\mathrm{S}}$ shells) ones. The signals vector becomes a concatenation of the signal vectors from different encodings, such as the LTE $\left(\mathbf{S}^{\mathrm{L}}\right)$, the PTE $\left(\mathbf{S}^{\mathrm{P}}\right)$ and the STE $\left(\mathbf{S}^{\mathrm{S}}\right)$ ones, leading to the following constrained linear least squares problem:

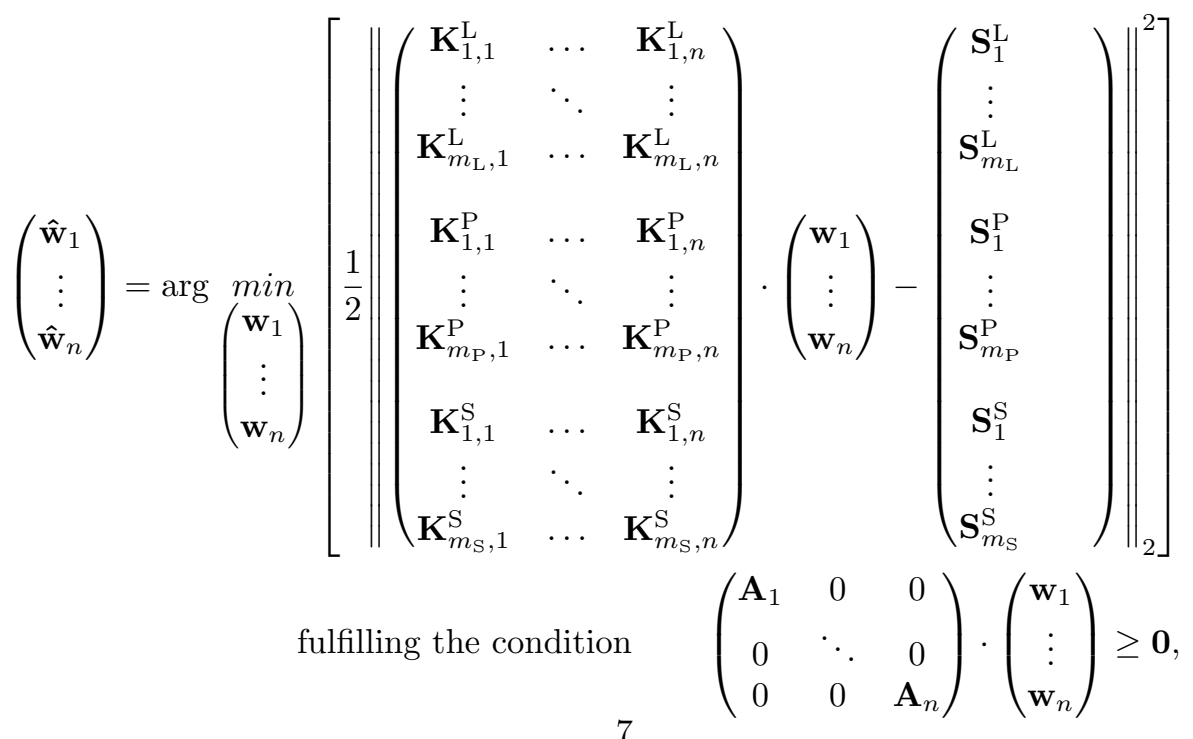


where $\mathbf{A}_{j}$ is the matrix relating the coefficients $\mathbf{w}_{j}$ to the fODF amplitudes. Again, solving this equation leads to the unknown vector of fODFs coefficients for each tissue. From these, the volume fraction (VF) of each tissue can be calculated as the amplitude of their first coefficient, leading to a sort of tissue classification.

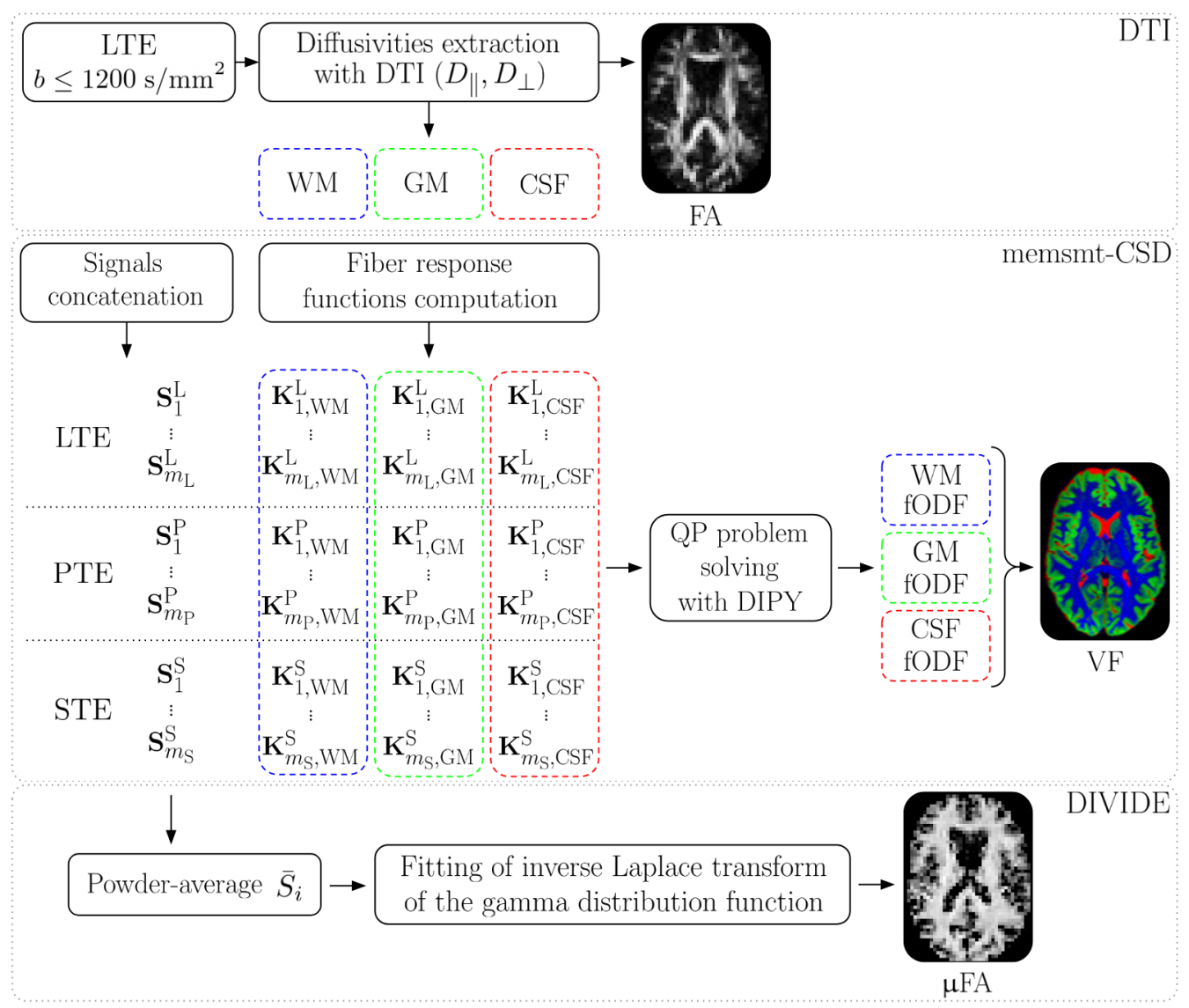

Figure 2: Summary of the computational steps used in this study, comprising DTI, memsmt-CSD and DIVIDE, for linear, planar and spherical tensor encodings, and three tissues (WM, GM and CSF). FA and $\mu$ FA images are adapted from Szczepankiewicz et al. [2015].

Both constrained linear least squares problems from equations 18 and 19 can be rewritten as a strictly convex quadratic programming $(\mathrm{QP})$ problem, using the compact nomenclature of equation 16 such that:

$$
\hat{\mathbf{w}}=\arg \min _{\mathbf{w}} \frac{1}{2} \mathbf{w}^{\mathrm{T}} \mathbf{P} \mathbf{w}+\mathbf{Q}^{\mathrm{T}} \mathbf{w} \quad \text { subject to } \mathbf{A} \mathbf{w} \geq \mathbf{0},
$$

where $\mathbf{P}=\mathbf{K}^{\mathrm{T}} \mathbf{K}$ and $\mathbf{Q}=-\mathbf{K}^{\mathrm{T}} \mathbf{S}$. This QP problem is solved with DIPY [Garyfallidis et al., 2014], which uses a QP solver implemented in CVXPY [Diamond and Boyd, 2016; Agrawal et al., 2018], based on the OSQP solver [Stellato et al., 2020]. The fiber response functions $K_{\epsilon_{i}}\left(b_{i} ; \mathbf{e}_{i}, \mathbf{u}\right)$ for each encoding and b-value pair are calculated from equation 
13 using diffusivities extracted from a DTI fit for each tissue. Figure 2 summarizes the computational steps of tensor-valued constrained spherical deconvolution.

\subsection{Measures computation}

The DTI measures such as FA and diffusivities (AD, MD, RD) are calculated using the eigenvalues of the diffusion tensor obtained from a DTI fit, as shown by Basser and Pierpaoli [1996]. The fit is performed only on the linear encoding data and with shells below $b=1200 \mathrm{~s} / \mathrm{mm}^{2}$.

The measures enabled by the combination of at least two different b-tensor encodings are obtained using the DIVIDE method. The implementation of this method is strongly inspired by Nilsson et al. [2018]. Note that although DIVIDE relies on powder-averaged signals, it has suggested to be, to some extent, robust to not perfectly rotation invariant signals [Reymbaut et al., 2020c]. The present study focuses on the microscopic fractional anisotropy ( $\mu \mathrm{FA}$ ) introduced by Lasič et al. [2014] and calculated using equation 7, in comparison to the FA. Figure 2 summarizes the processing steps leading to the FA and $\mu \mathrm{FA}$.

\subsection{Simulating tensor-valued diffusion data}

To study the impact of different combination of diffusion encoding shapes and number of directions per shells on the fODFs and the computation of the $\mu \mathrm{FA}$, tensor-valued diffusion data is simulated using a discrete version of equation 1 . The first step is to choose a set of $N_{k}$ diffusion tensors $\mathbf{D}_{k}$ that will describe the composition of the voxel, each of them being associated with a tissue compartment. These diffusion tensors are defined by their eigenvalues in their principal axis system (PAS), such that

$$
\mathbf{D}_{k, \text { PAS }}=\left(\begin{array}{ccc}
D_{k, \|} & 0 & 0 \\
0 & D_{k, \perp} & 0 \\
0 & 0 & D_{k, \perp}
\end{array}\right)
$$

where $D_{k, \|}$ and $D_{k, \perp}$ are the axial and radial diffusivities, respectively. For each of these diffusion tensors, $D_{k, \|}$ and $D_{k, \perp}$ are converted into the isotropic diffusivity $D_{k \text {,iso }}$ and the normalized anisotropy $D_{k, \Delta}$ [Conturo et al., 1996], describing the anisotropy of the diffusion tensor, following the relations $D_{k \text {,iso }}=\left(D_{k, \|}+2 D_{k, \perp}\right) / 3$ and $D_{k, \Delta}=$ $\left(D_{k, \|}-D_{k, \perp}\right) /\left(3 D_{k \text {,iso }}\right)$. Then, both $D_{k \text {,iso }}$ and $D_{k, \Delta}$ become the mean of discrete Gaussian distributions with a given relative standard deviation (STD) $\sigma_{k}$ (relative to its mean) and $N$ discrete elements. These two Gaussian distributions are weighted by the volume fraction and the non-DW signal $S_{0_{k}}$ of the tissue compartment they represent. The distribution centered at $D_{k \text {,iso }}$ is also flipped to ensure a negative covariance between the two distributions. Then, the distributions of $D_{k \text {, iso }}$ and $D_{k, \Delta}$ are converted back to $D_{k, \|}$ and $D_{k, \perp}$ distributions, creating a distribution of diffusion tensors $P_{\mathbf{D}_{k}}$ described by the distributions of axial and radial diffusivities. Every diffusion tensor in the DTD is rotated by given angles $\theta_{k}$ and $\phi_{k}$, corresponding to the orientation of their initial mean diffusion tensor $\mathbf{D}_{k}$. The angles $\theta_{k}$ and $\phi_{k}$ follow the physics convention for spherical coordinates, meaning that $\theta_{k}$ gives the angle with respect to the $z$ axis, while $\phi_{k}$ gives the angle with respect to the $x$ axis. Finally, the $N_{k}$ weighted DTD are concatenated into a single DTD $P_{\mathbf{D}_{t}}$ representing the whole voxel's content with $N_{t}=N_{k} \cdot N$ diffusion 
tensors $\mathbf{D}_{t}$. This allows the computation, for any chosen b-tensor $\mathbf{B}_{i}$, of the diffusion signal in the voxel:

$$
S_{i}=\sum_{t=1}^{N_{t}} P_{\mathbf{D}_{t}} e^{-\mathbf{B}_{i}: \mathbf{D}_{t}} .
$$

The signal obtained from this process can also be subject to added noise, which is calculated with respect to a given $S_{0}$ and signal to noise ratio (SNR), relative the chosen $S_{0}$ :

$$
S=S_{0} \sqrt{\left(\frac{S}{S_{0}}+\frac{1}{\mathrm{SNR}} \cdot \sigma_{\text {rand }}\right)^{2}+\left(\frac{1}{\mathrm{SNR}} \cdot \sigma_{\text {rand }}\right)^{2}},
$$

where $\sigma_{\text {rand }}$ is a random number going from 0 to 1 .

From the DTD, the ground truth $\mu \mathrm{FA}$ is calculated using equations from section 2.1.

\subsection{Simulated anatomy}

The simulation method previously described is used to generate the DW signals of five typical voxels, representing the fictional anatomy of this study. These voxels are composed of various volume fractions of four different tissue compartments $\left(N_{k}=4\right)$, each compartment $k$ being defined by the parameters $D_{k, \|}, D_{k, \perp}, \theta_{k}, \phi_{k}, \sigma_{k}$ and $S_{0, k}$, with $N=100$ for each of them. Table 1 shows the parameters configuration for each tissue compartment, comprising two identical WM compartments separated by a certain angle $\alpha$, one grey matter (GM) compartment and one cerebrospinal fluid (CSF) compartment. Table 2 presents the composition of the five simulated voxels. The first voxel is a WM fiber crossing of equal proportions, described by the separation angle $\alpha$. The second voxel is a single WM fiber, while the fourth and fifth voxels are 100\% GM and CSF, respectively. The third voxel represents a partial volume between voxel 2 and voxel 4 , composed of $50 \%$ WM fiber and 50\% GM.

\begin{tabular}{||c|cccc||}
\hline Compartments & $\mathrm{D}_{k, \|}\left(\times 10^{-3} \mathrm{~mm}^{2} / \mathrm{s}\right)$ & $\mathrm{D}_{k, \perp}\left(\times 10^{-3} \mathrm{~mm}^{2} / \mathrm{s}\right)$ & $\theta_{k}$ & $\mathrm{~S}_{0, k}$ \\
\hline \hline WM1 & 1.7 & 0.3 & 90 & 1100 \\
WM2 & 1.7 & 0.3 & $90-\alpha$ & 1100 \\
GM & 0.6 & 0.6 & 0 & 1500 \\
CSF & 3.0 & 3.0 & 0 & 3700 \\
\hline
\end{tabular}

Table 1: Parameters configuration of the four tissue compartments used to generate simulated data. The two WM compartments are built from the same diffusivities and only differ by the orientation of their diffusion tensor $\mathbf{D}_{k}$, separated by $\alpha$ degrees in the $y-z$ plane. The angle $\theta_{k}$ for GM and CSF are both equal to 0 since their diffusion tensor is isotropic, thus not depending on orientation. For all compartments, $\phi_{k}=0$ and $\sigma_{k}=0.15$. A different non-DW signal $S_{0, k}$ is set for each tissue type.

The choice of the axial and radial diffusivities is motivated by typical in vivo diffusivity values. WM and CSF diffusivities are inspired by Pierpaoli and Basser [1996]; Alexander [2008]; Alexander et al. [2010]; Zhang et al. [2012], while GM diffusivity value comes from Pierpaoli and Basser [1996]; Liu et al. [2006]. Moreover, the non-DW signal $S_{0, k}$ set for WM, GM and CSF are approximated from data obtained from the MGH-USC Human Connectome Project database [Glasser et al., 2013; Sotiropoulos et al., 2013]. 


\begin{tabular}{||c|ccccc||}
\hline Compartments & Voxel 1 & Voxel 2 & Voxel 3 & Voxel 4 & Voxel 5 \\
\hline \hline WM1 & 50 & 100 & 50 & 0 & 0 \\
WM2 & 50 & 0 & 0 & 0 & 0 \\
GM & 0 & 0 & 50 & 100 & 0 \\
CSF & 0 & 0 & 0 & 0 & 100 \\
\hline
\end{tabular}

Table 2: Voxels composition, described by the volume fractions, in percentage, of each tissue compartment.

Throughout the simulation experiments, only the angle $\alpha$ and the SNR vary, as does the set of b-tensors $\mathbf{B}_{i}$. The relative STD $\sigma_{k}$ stays constant at $15 \%$, providing some variance to the distribution of diffusion tensors composing the voxels. To test the limits of memsmt-CSD, the WM fiber crossing of voxel 1 is simulated many times using different values of $\alpha$, from 90 to 50 degrees. The data is simulated without noise $(\mathrm{SNR}=\infty)$ and with $\mathrm{SNR}=30, \mathrm{SNR}=20$ and $\mathrm{SNR}=15$, inspired from in vivo values of data discussed in later sections. These allow to study the effects of noise on the memsmt-CSD and the DIVIDE processes, as well as the effects of spatial resolution and echo time (TE). Indeed, higher spatial resolution or shorter TE can be mimicked by a higher SNR, for example by comparing $\mathrm{SNR}=20$ to $\mathrm{SNR}=15$.

\subsection{Simulated datasets}

The fictional anatomy set up is used to explore and test different acquisition protocols, each represented by a set of b-tensors $\mathbf{B}_{i}$. These schemes can also be described by a set of b-values, a number of diffusion encoding gradient directions and different choices of encoding shapes. This representation is favoured over the b-tensor itself, as it is easier to grasp and compare to conventional acquisition schemes. The chosen b-values and number of gradient directions per shell, adapted from Nilsson et al. [2020] to allow memsmtCSD, are presented in table 3 as $\mathrm{L}, \mathrm{P}_{1}, \mathrm{P}_{2}, \mathrm{~S}_{1}$ and $\mathrm{S}_{2}$. A typical multi-shell multi-tissue acquisition [Theaud et al., 2020] is also added as $\mathrm{L}_{\mathrm{msmt}}$ for comparison purposes, as it contains the same total amount of directions as $\mathrm{L}$ and $\mathrm{S}_{2}$ combined.

\begin{tabular}{||c|cccccc||}
\hline $\begin{array}{c}\text { b-value } \\
\left(\mathrm{s} / \mathrm{mm}^{2}\right)\end{array}$ & $\begin{array}{c}\text { Linear }_{1} \\
(\mathrm{~L})\end{array}$ & $\begin{array}{c}\text { Linear }_{2} \\
\left(\mathrm{~L}_{\mathrm{msmt}}\right)\end{array}$ & $\begin{array}{c}\text { Planar }_{1} \\
\left(\mathrm{P}_{1}\right)\end{array}$ & $\begin{array}{c}\text { Planar }_{2} \\
\left(\mathrm{P}_{2}\right)\end{array}$ & $\begin{array}{c}\text { Spherical }_{1} \\
\left(\mathrm{~S}_{1}\right)\end{array}$ & $\begin{array}{c}\text { Spherical } \\
\left(\mathrm{S}_{2}\right)\end{array}$ \\
\hline \hline 0 & 3 & 5 & 1 & 2 & 1 & 2 \\
100 & 3 & 0 & 3 & 6 & 3 & 6 \\
700 & 3 & 8 & 3 & 6 & 3 & 6 \\
1200 & 12 & 30 & 6 & 10 & 6 & 10 \\
1800 & 18 & 0 & 6 & 16 & 6 & 16 \\
2400 & 24 & 60 & 0 & 0 & 0 & 0 \\
\hline \hline Total & 63 & 103 & 19 & 40 & 19 & 40 \\
\hline
\end{tabular}

Table 3: Six gradient tables chosen for this study. Each column shows the number of encoding directions in the corresponding shell. The last row shows the total number of directions, including $b=0 \mathrm{~s} / \mathrm{mm}^{2}$.

The encoding directions are distributed between shells and encodings using the elec- 
trostatic repulsion implementation of Caruyer et al. [2013] (with default inter-shell coupling weights). More precisely, the $\mathrm{L}$ and $\mathrm{L}_{\mathrm{msmt}}$ directions are first both calculated separately from the other encodings. Then, the directions are recalculated when $\mathrm{L}$ is used in a protocol with planar encoding, to get the best directional coverage over the shells and the encodings. Directional considerations are different for spherical encoding, as the framework laid out in Section 2 presents spherical diffusion encoding as a perfectly isotropic measurement. In practice, anisotropy in the frequency content of spherically encoded gradient waveforms [Lundell and Lasič, 2020; Szczepankiewicz et al., 2020] and eddy currents [Szczepankiewicz et al., 2020] create situations wherein spherical b-tensors should preferably be rotated. This means that there is a set of directions for $\mathrm{L}$ when it is alone or with spherical encoding only, and different sets of directions when it is combined with $\mathrm{P}_{1}$ or $\mathrm{P}_{2}$, which we call $\mathrm{L}^{*}$ and $\mathrm{L}^{* *}$, respectively. The spherical encoding directions from $\mathrm{S}_{2}$ are also separately calculated using the same method, and the directions of $S_{1}$ are subsampled from it. The subsampling of the encoding directions is done using a method that chooses the directions such as to minimize the electrostatic repulsion energy, based on the implementation from Caruyer et al. [2013]. All of these gradient directions distributions are shown and available at figure A.14.

The gradient tables described in table 3 are combined in different ways to test the impact of diffusion encodings and number of encoding directions on both memsmt-CSD and DIVIDE, while varying the $\alpha$ angle and the SNR. The tested protocols are presented in table 4 . Note that we add a separate protocol, $\mathrm{L}_{\mathrm{msmt}}$, corresponding to the gradient table $\mathrm{L}_{\mathrm{msmt}}$ and only tested with memsmt-CSD.

\begin{tabular}{||cccccccc||}
\hline $\mathrm{L}$ & $\mathrm{LP}_{1}$ & $\mathrm{LP}_{2}$ & $\mathrm{LS}_{1}$ & $\mathrm{LS}_{2}$ & $\mathrm{LP}_{1} \mathrm{~S}_{1}$ & $\mathrm{LP}_{2} \mathrm{~S}_{1}$ & $\mathrm{LP}_{2} \mathrm{~S}_{2}$ \\
\hline \hline $\mathrm{L}$ & $\mathrm{L}^{*}, P_{1}$ & $\mathrm{~L}^{* *}, P_{2}$ & $\mathrm{~L}, \mathrm{~S}_{1}$ & $\mathrm{~L}, \mathrm{~S}_{2}$ & $\mathrm{~L}^{*}, P_{1}, \mathrm{~S}_{1}$ & $\mathrm{~L}^{* *}, P_{2}, \mathrm{~S}_{1}$ & $\mathrm{~L}^{* *}, P_{2}, \mathrm{~S}_{2}$ \\
\hline
\end{tabular}

Table 4: Eight protocols tested in the study (first row). These are combinations of gradient tables from table 3 (second row). Note that the set of directions for L changes depending on whether or not it is combined with either of the planar encoding gradient tables. These linear encoding directions sets are defined as $\mathrm{L}$ (without planar), $\mathrm{L}^{*}$ (with $\mathrm{P}_{1}$ ) and $\mathrm{L}^{* *}$ (with $\mathrm{P}_{2}$ ).

Each of these protocols are simulated $N_{\text {rep }}=1000$ times, to avoid outliers due to noise. All the outputs of memsmt-CSD, the DTI fit and DIVIDE are averaged over the $N_{\text {rep }}$ repetitions. Their standard deviation allows to keep track of the variability of the process and compute the precision of it.

\subsection{In vivo acquisitions}

To confirm the conclusions drawn from the simulated data, protocol $\mathrm{LS}_{2}$ was acquired in vivo on two healthy male volunteers. These in vivo data acquisitions were taken on a Philips Ingenia 3T system with a 32-channel head coil (Philips Healthcare, Best, The Netherlands). Tensor-valued dMRI was made possible by a prototype spin-echo singleshot EPI sequence that enables the use of arbitrary b-tensor shapes for diffusion encoding. Planar and spherical encoding were achieved using asymmetric gradient waveforms, optimized to minimize TE using a constrained optimization method described in Sjölund et al. [2015], with the following settings: Euclidian norm, heat dissipation factor 0.7, amplitude limit of $45 \mathrm{mT} / \mathrm{m}$ and a slew rate limit of $90 \mathrm{~T} / \mathrm{m} / \mathrm{s}$. Imaging was performed on the two volunteers at different spatial resolutions, with the following set of constant 
parameters: $\mathrm{SENSE}=2$, Multiband-SENSE $=2$, and partial-Fourier $=0.65$, and two sets of parameters depending on the resolution. The set at $2.5 \mathrm{~mm}$ isotropic (first volunteer) was: resolution $=2.5 \times 2.5 \times 2.5 \mathrm{~mm}^{3}, \mathrm{TE}=117 \mathrm{~ms}, \mathrm{TR}=5.6 \mathrm{~s}, \mathrm{FOV}=240 \times 240$ $\mathrm{mm}^{2}$, slices $=48$, while the set at $2.0 \mathrm{~mm}$ isotropic (second volunteer) was: resolution $=2 \times 2 \times 2 \mathrm{~mm}^{3}, \mathrm{TE}=119.5 \mathrm{~ms}, \mathrm{TR}=7.2 \mathrm{~s}, \mathrm{FOV}=224 \times 224 \mathrm{~mm}^{2}$, slices $=60$. Given these sets of parameters, the acquisition times that would have each protocol from table 4 are presented in table 5 . Note that the significant increase of time compared to the 3 minutes protocol of Nilsson et al. [2020] is due to the added number of directions for LTE, the different MRI scanner and the increased resolution for the set at $2 \mathrm{~mm}$ isotropic.

To get a comparative view of the performances of protocol $\mathrm{LS}_{2}$, the typical multishell multi-tissue protocol $\mathrm{L}_{\mathrm{msmt}}$ was also acquired on the second volunteer, at a spatial resolution of $2.0 \mathrm{~mm}$ isotropic. Since the encoding shape has a major impact on gradient waveforms optimization, leading to longer TE for STE then for LTE or PTE, this protocol was tested with two different echo times. Thus, the TE took values of $119.5 \mathrm{~ms}$ (same as $\mathrm{LS}_{2}$ ) and $86 \mathrm{~ms}$, while keeping the same $\mathrm{TR}=7.2$ and other parameters, meaning that protocol $\mathrm{L}_{\mathrm{msmt}}$ has the same acquisition time as protocol $\mathrm{LS}_{2}$. Furthermore, the spatial resolutions and echo times chosen for the in vivo acquisitions justify the SNR values described in section 3.4. Indeed, the difference in SNR between the $2.5 \mathrm{~mm}$ isotropic and $2.0 \mathrm{~mm}$ isotropic acquisitions should be given by a ratio of approximately 2 , as $2.5^{3} / 2^{3}=1.95$, meaning that simulations with $\mathrm{SNR}=30$ and $\mathrm{SNR}=15$ can be used to study the change in resolution. As for changes in TE, simulations with $\mathrm{SNR}=15$ and $\mathrm{SNR}=20$ are suited to compare $\mathrm{TE}=119.5 \mathrm{~ms}$ with $\mathrm{TE}=86 \mathrm{~ms}$.

\begin{tabular}{||ccccccccc||}
\hline & $\mathrm{L}$ & $\mathrm{LP}_{1}$ & $\mathrm{LP}_{2}$ & $\mathrm{LS}_{1}$ & $\mathrm{LS}_{2}$ & $\mathrm{LP}_{1} \mathrm{~S}_{1}$ & $\mathrm{LP}_{2} \mathrm{~S}_{1}$ & $\mathrm{LP}_{2} \mathrm{~S}_{2}$ \\
\hline \hline Set 2.5 mm iso & $6: 22$ & $8: 26$ & $10: 29$ & $8: 26$ & $10: 29$ & $10: 30$ & $12: 33$ & $14: 36$ \\
Set 2.0 mm iso & $8: 11$ & $10: 50$ & $13: 29$ & $10: 50$ & $13: 29$ & $13: 29$ & $16: 08$ & $18: 47$ \\
\hline
\end{tabular}

Table 5: Real acquisition times of the protocols presented in table 4, in minutes.

$\mathrm{T}_{1}$-weighted images were also acquired at a resolution of $0.8 \times 0.8 \times 0.8 \mathrm{~mm}^{3}$ to help identifying the tissue types for the computation of the response functions.

\subsection{Processing}

The in vivo DW data was preprocessed using an adapted version of the Tractoflow pipeline [Theaud et al., 2020]. More precisely, the pipeline performs the MP-PCA denoising technique (Mrtrix3) [Veraart et al., 2016], followed by susceptibility-induced distortion correction (FSL topup) [Andersson et al., 2003; Smith et al., 2004] and N4 bias field correction (ANTs) [Avants et al., 2009], on all DW images. Motion correction was not necessary on these less than 15 min long acquisitions.

The $\mathrm{T}_{1}$-weighted images were also treated by the pipeline, starting with a brain extraction tool (BET) [Smith, 2002] from FSL and a non-local means denoising (DIPY) [Coupé et al., 2008]. Then, the structural images were segmented into three tissues, WM, GM and CSF, using the FAST tool [Zhang et al., 2001] from FSL and finally registered on the DW images with ANTs. 

made available under aCC-BY 4.0 International license.

This tissue segmentation led to binary masks necessary to extract axial and radial diffusivities for each tissue using a DTI model fitted with a weighted least squares method. The memsmt-CSD method was computed with a maximal SH order of 8 and with the Descoteaux07 [Descoteaux et al., 2007] SH basis adapted by DIPY, and using the default parameters of OSQP. As for the fit of the inverse Laplace transform of the gamma distribution function, the set of parameters were, following the nomenclature of [Nilsson et al., 2018]: do_weight=True, do_pa_weight=True, do_multiple_s0=True, fit_iters=1, guess_iters $=50$ and all other default parameters from the authors.

\section{Results}

\subsection{Simulated data}
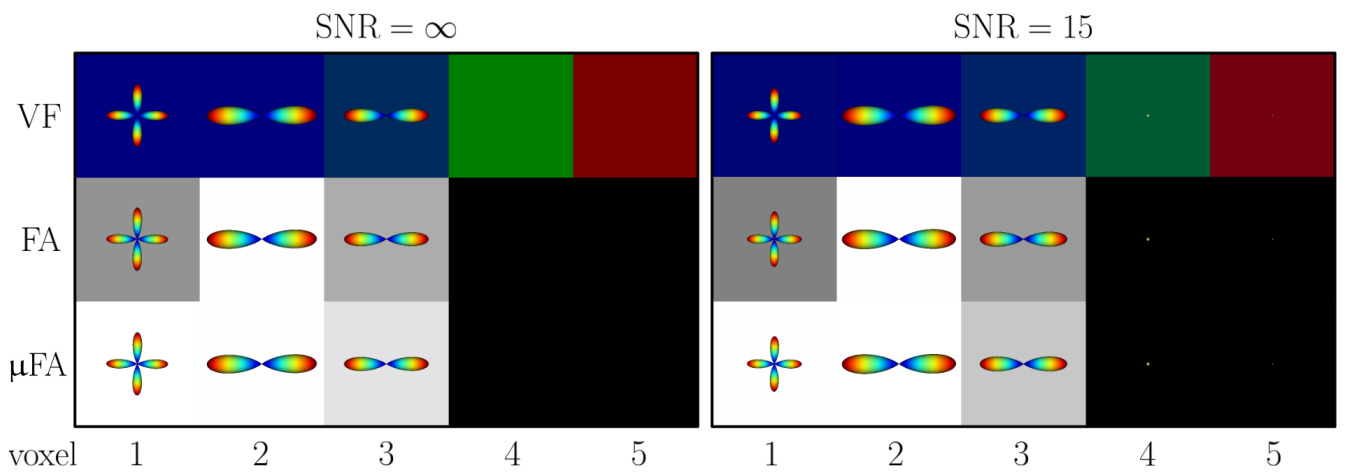

Figure 3: Demonstration of memsmt-CSD, DTI fit and DIVIDE methods on the simulated anatomy described in section 3.4, with protocol $\mathrm{LP}_{2} \mathrm{~S}_{2}$ and a separation angle of 90 degrees in voxel 1 , for $\mathrm{SNR}=\infty$ and 15. The first row is the memsmt-CSD output, namely the WM fODFs and the volume fractions, RGB coded. The second and third rows show the FA calculated from a DTI fit and the $\mu$ FA computed from DIVIDE, respectively. The fODFs are added on top of them to emphasize the contents of the voxels. FA and $\mu \mathrm{FA}$ values go from 0 to 1 (black to white color gradient).

Figure 3 shows an example of the memsmt-CSD, DTI fit and DIVIDE outputs from the fictional anatomy described in section 3.4, using simulated protocol $\mathrm{LP}_{2} \mathrm{~S}_{2}$ with a crossing of 90 degrees, without noise and with $\mathrm{SNR}=15$. More precisely, it displays the fODFs obtained from memsmt-CSD on top of the derived volume fractions. The VF are represented with an RGB code, where red, green and blue are the CSF, GM and WM channels, respectively. For each voxel, the VF is normalized by the maximum value of all voxels, meaning that the VF keeps track of the absolute amplitude of each channel. A voxel containing partial volumes will therefore appear as a darker mix of the implicated channel, as seen in voxel 3 with the darker blue color (50\% WM and 50\% GM). Note that all VF voxels in this work are displayed with an opacity factor of 0.5 as well, with the purpose of increasing the fODFs visibility. Figure 3 also shows a comparison between the FA calculated from DTI and the $\mu \mathrm{FA}$ extracted with the DIVIDE method, with values comprised between 0 (black) to 1 (white). 
bioRxiv preprint doi: https://doi.org/10.1101/2021.04.07.438845; this version posted May 25, 2021. The copyright holder for this preprint (which was not certified by peer review) is the author/funder, who has granted bioRxiv a license to display the preprint in perpetuity. It is made available under aCC-BY 4.0 International license.

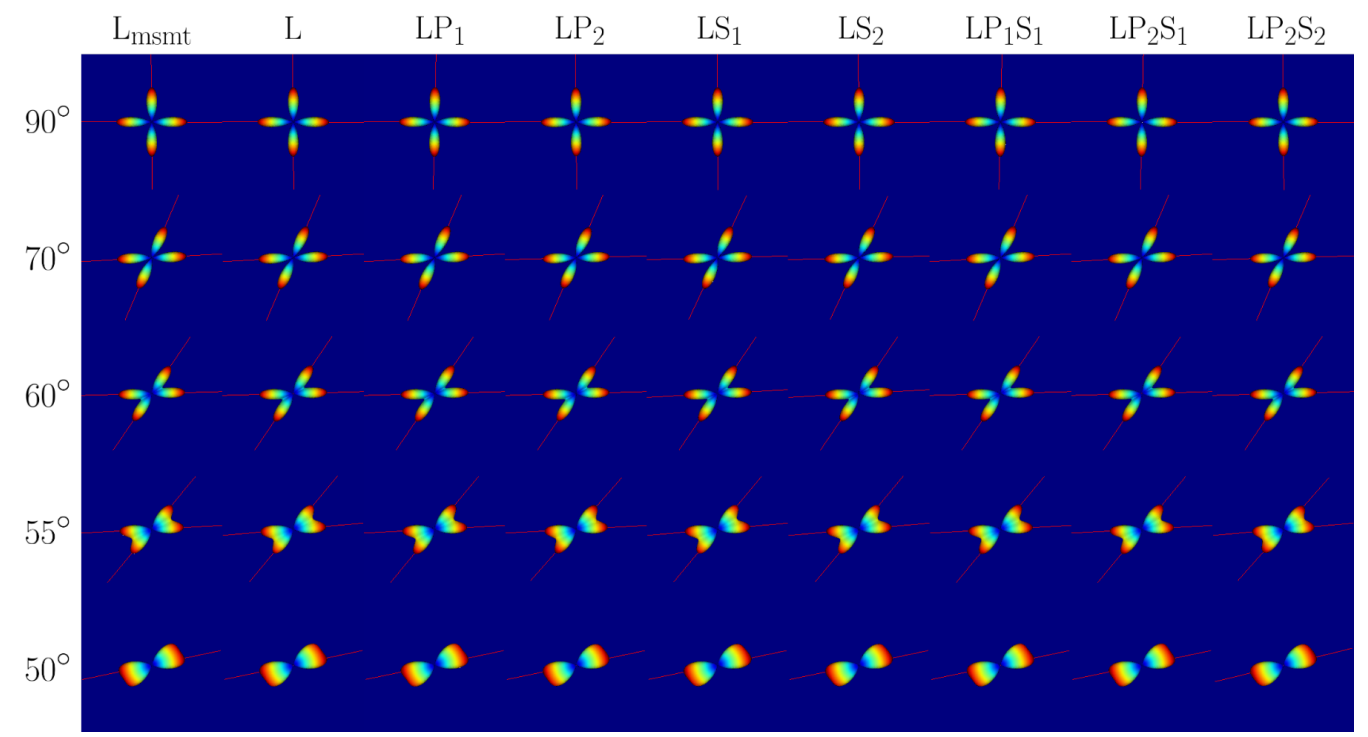

Figure 4: Crossing fibers of voxel 1 reconstructed with memsmt-CSD from the protocols presented in table 4 and protocol $\mathrm{L}_{\mathrm{msmt}}$, at various separation angles and for $\mathrm{SNR}=\infty$. In the background is the associated VF map, which is $100 \% \mathrm{WM}$ and thus blue in this case. The red lines are the detected maxima of the fODFs.

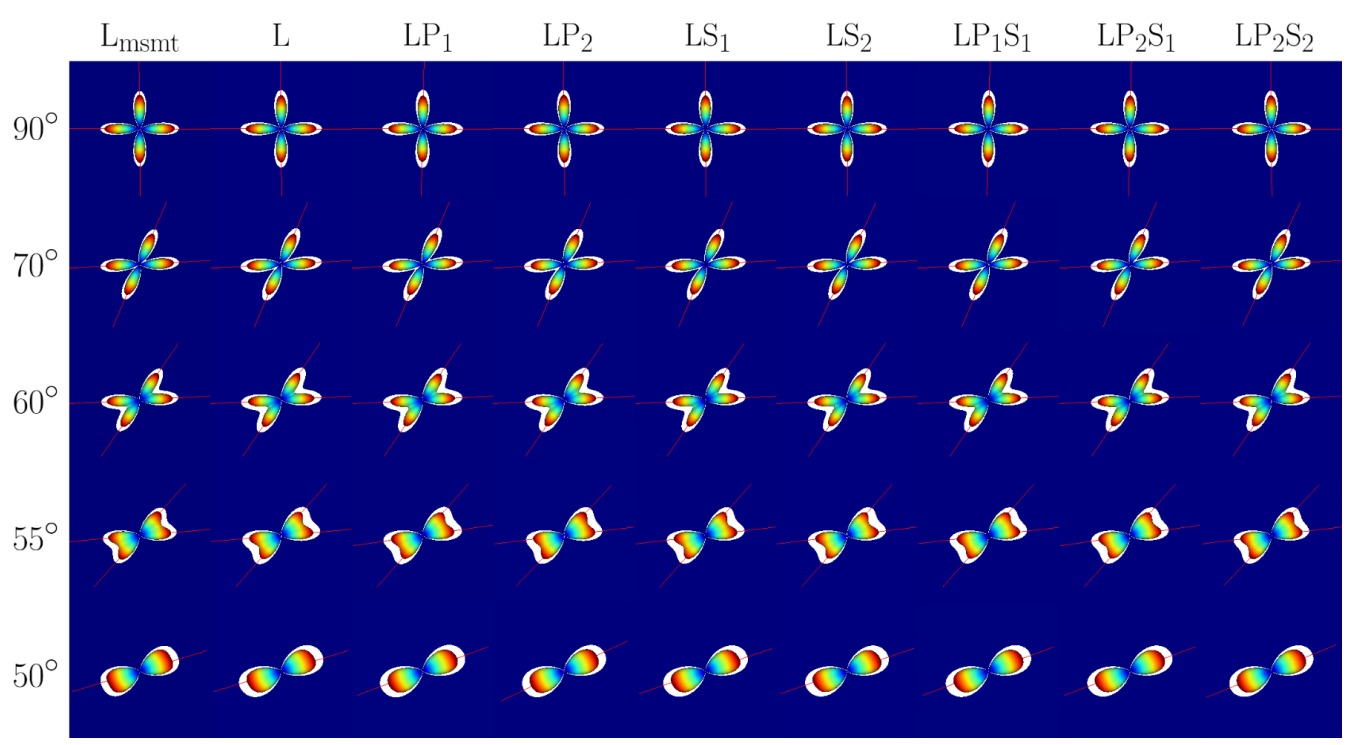

Figure 5: Crossing fibers of voxel 1 reconstructed with memsmt-CSD from the protocols presented in table 4 and protocol $\mathrm{L}_{\mathrm{msmt}}$, at various separation angles and for $\mathrm{SNR}=30$. In the background is the associated VF map, which is $100 \% \mathrm{WM}$ and thus blue in this case. The colored surfaces correspond to the mean fODF over 1000 simulations, whereas the white surfaces represent the mean plus two standard deviations. The red lines are the detected maxima of the fODFs. 


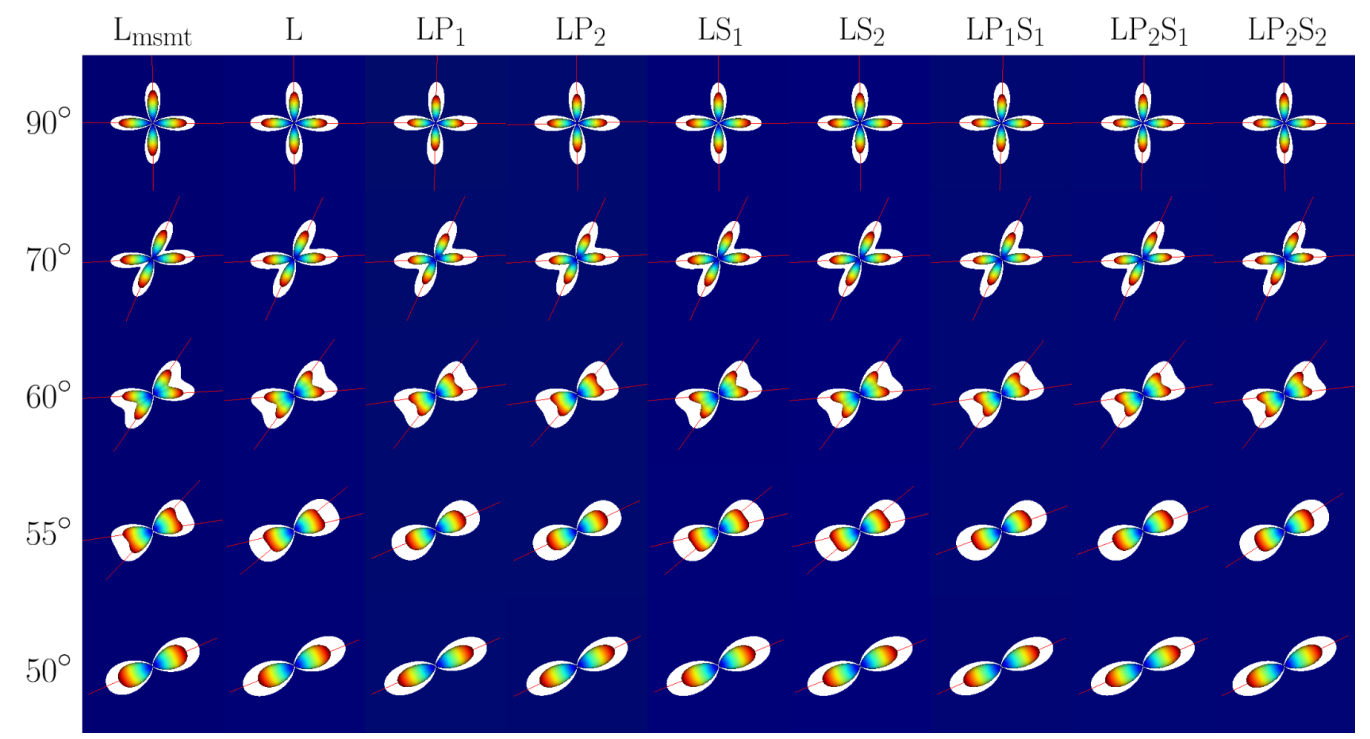

Figure 6: Crossing fibers of voxel 1 reconstructed with memsmt-CSD from the protocols presented in table 4 and protocol $\mathrm{L}_{\mathrm{msmt}}$, at various separation angles and for $\mathrm{SNR}=15$. In the background is the associated VF map, which is 100\% WM and thus blue in this case. The colored surfaces correspond to the mean fODF over 1000 simulations, whereas the white surfaces represent the mean plus two standard deviations. The red lines are the detected maxima of the fODFs.

The crossing WM fibers separation performances of each protocol from table 4 and protocol $\mathrm{L}_{\mathrm{msmt}}$ are shown on figures 4,5 and 6 for $\mathrm{SNR}=\infty, \mathrm{SNR}=30$ and $\mathrm{SNR}=15$, respectively. The WM fODFs are the mean of 1000 repetitions of the simulation for $\mathrm{SNR}=15$ and $\mathrm{SNR}=30$, and the variance of this fODFs distribution is visible as the white lobes around the fODFs, corresponding precisely to the fODFs times two standard deviations. Without noise, all protocols show a very similar performance, being able to separate the two fibers up to an angle of 55 degrees and failing for 50 degrees. The same outcome is visible at $\mathrm{SNR}=30$.

As for $\mathrm{SNR}=15$, each protocol is able to separate the two peaks up to an angle of 60 degrees, but protocols containing planar tensor encoding, $\mathrm{P}_{1}$ or $\mathrm{P}_{2}$, fail for tighter angles. The linear tensor only protocol (L), the typical multi-shell multi-tissue protocol $\mathrm{L}_{\mathrm{msmt}}$ and the combined linear and spherical tensor encodings protocols $\left(\mathrm{LS}_{1}\right.$ and $\left.\mathrm{LS}_{2}\right)$ achieve peaks separation at 55 degrees. Furthermore, these protocols seem to produce tighter fODFs, especially $\mathrm{L}_{\mathrm{msmt}}$. Each protocol performs better with less noise in terms of angular resolution and precision, according to the diminishing variance of the fODFs.

Figure 7 shows a more precise representation of the angular resolution of the tested protocols at every studied SNR values, through a plot of the number of fiber orientations (NuFO) extracted with respect to the separation angle. This points out the exact angle at which the protocols fail to separate the two fibers, noticeable by the drops in the curves, allowing a better understanding of the behaviours presented on figures 4,5 and 6.

Without noise, all protocols perform the same, losing the WM fiber crossing right before the 50 degrees mark, at 51 degrees, as the NuFO drops from 2 to 1 . At $\mathrm{SNR}=30$, protocols $\mathrm{L}, \mathrm{L}_{\mathrm{msmt}}, \mathrm{LS}_{2}$ and $\mathrm{LS}_{1}$ start to separate themselves from protocols containing 

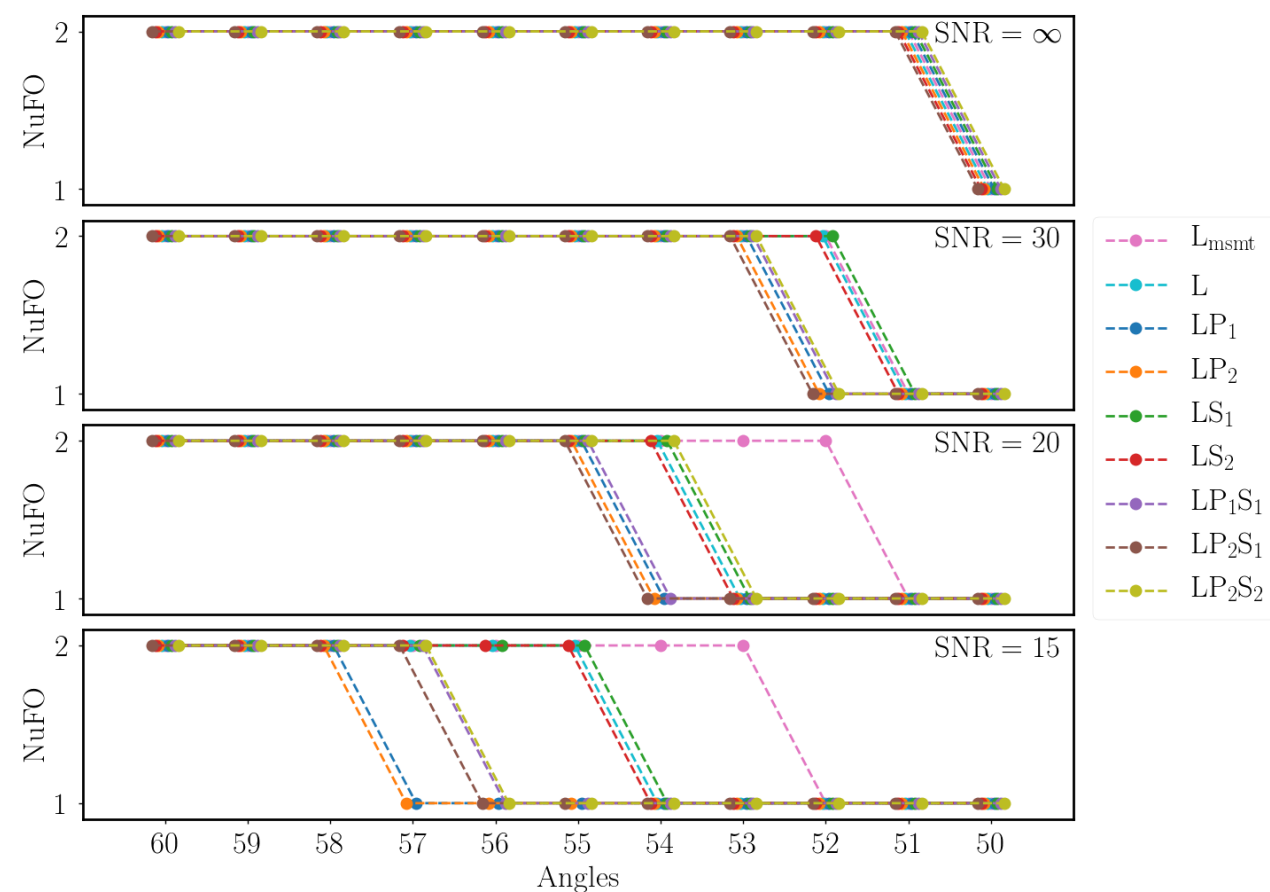

Figure 7: Angular dependency of the number of fiber orientations extracted from the mean fODF over 1000 simulations with memsmt-CSD from the studied protocols in voxel 1, at various SNR. Note that for each angle, the dots are slightly shifted to better distinguish the protocols, but touching dots are truly at the same angle value.

planar tensor encoding $\left(\mathrm{P}_{1}\right.$ or $\left.\mathrm{P}_{2}\right)$, as they lose 1 degree from $\mathrm{SNR}=\infty$, whereas all the other protocols lose 2 degrees. Protocol $\mathrm{L}_{\mathrm{msmt}}$ remains the same at $\mathrm{SNR}=20$, distancing itself from protocols $\mathrm{L}, \mathrm{LS}_{2}$ and $\mathrm{LS}_{1}$, which drop from 52 to 54 degrees of angular resolution. At this SNR level, all protocols with PTE also drop by 2 degrees ( 55 degrees), except for $\mathrm{LP}_{2} \mathrm{~S}_{2}$, which has an angular resolution of 54 degrees. While figure 6 shows a seemingly big difference ( 5 degrees) between $\mathrm{L}, \mathrm{L}_{\mathrm{msmt}}, \mathrm{LS}_{2}$ and $\mathrm{LS}_{1}$ and the rest of the protocols at $\mathrm{SNR}=15$, figure 7 indicates that all protocols differ from a maximum of 3 degrees at this $\mathrm{SNR}$, with the exception of $\mathrm{L}_{\mathrm{msmt}}$. Indeed, the combination of only linear and planar encodings $\left(\mathrm{LP}_{2}\right.$ and $\left.\mathrm{LP}_{1}\right)$ has the lowest angular resolution at 58 degrees, followed closely by the protocols composed of all encoding types $\left(\mathrm{LP}_{1} \mathrm{~S}_{1}, \mathrm{LP}_{2} \mathrm{~S}_{1}\right.$ and $\mathrm{LP}_{2} \mathrm{~S}_{2}$ ) with 57 degrees. The $\mathrm{L}, \mathrm{LS}_{2}$ and $\mathrm{LS}_{1}$ protocols are able to distinguish tighter angles, dropping from 2 to $1 \mathrm{NuFO}$ after 55 degrees. The $\mathrm{L}_{\text {msmt }}$ protocol has the highest angular resolution with 53 degrees. Note that protocol $\mathrm{LP}_{2}$ was also tested using the $\mathrm{L}$ gradient table instead of the $\mathrm{L}^{* *}$ gradient table to make sure that the directions redistribution does not impact the angular resolution of memsmt-CSD. Since the results are exactly the same as the normal $\mathrm{LP}_{2}$, the points are not shown on figure 7 .

The precision and accuracy of the DIVIDE method at computing the $\mu \mathrm{FA}$ with different protocols is presented on figure 8 , where the $\mu \mathrm{FA}$ from every protocol, as well as the ground truth $\mu \mathrm{FA}$, are plotted for the five simulated voxels, for each SNR. Throughout 


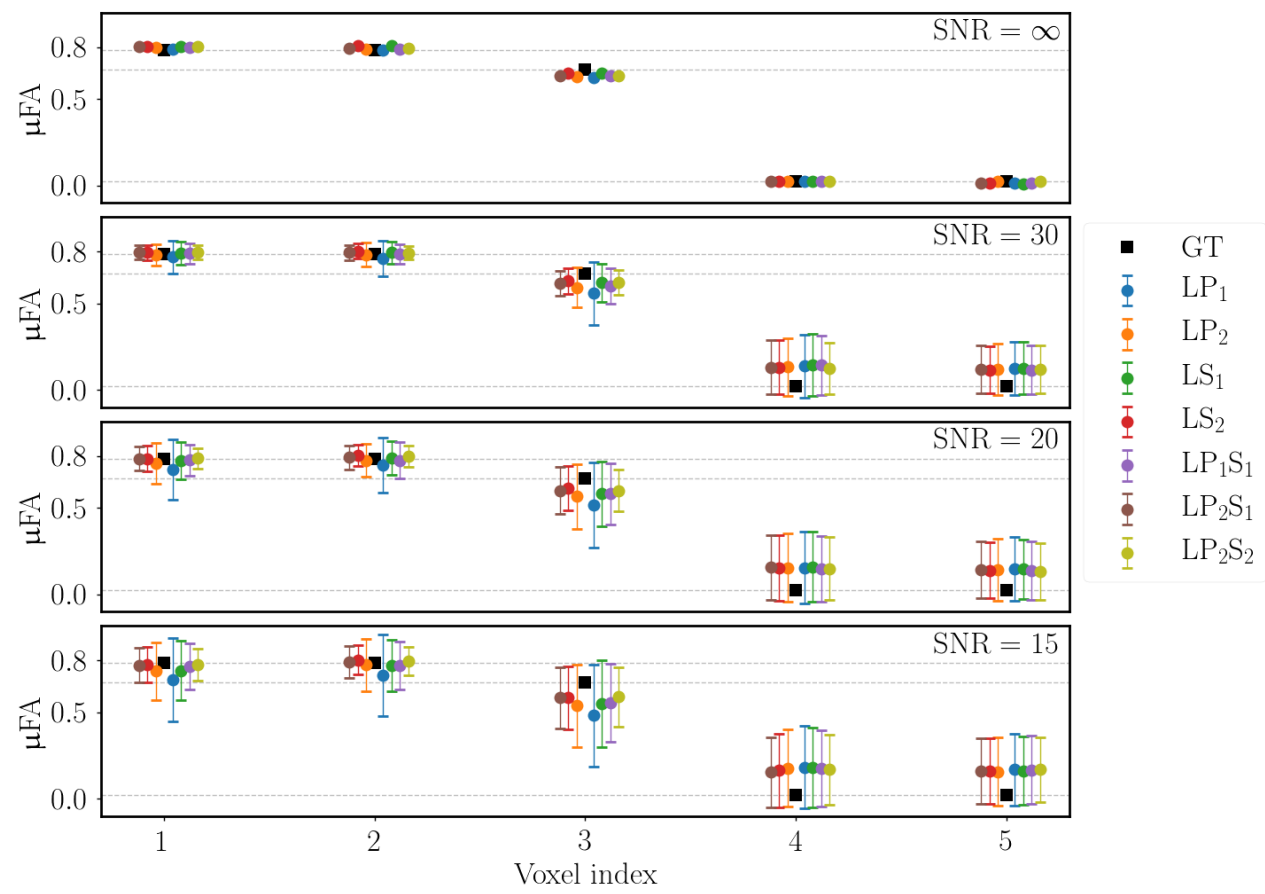

Figure 8: Mean $\mu$ FA over 1000 simulations for each voxel and each protocols, at various SNR. The error bars correspond to the standard deviation extracted from the 1000 repetitions of the simulations. The black squares and the horizontal dashed grey lines represent the ground truth $\mu \mathrm{FA}$.

each protocol, the $\mu$ FA computed without noise is following the ground truth value, while the noisy data deviates from it. The error bars, computed from the variance over the 1000 repetitions, also show the precision of the protocols. It is worth noting that the $\mu \mathrm{FA}$ computed for every protocol at each voxel is always overlapping with the ground truth value when considering the error bars, for both $\mathrm{SNR}=15,20$ and 30. For voxels 1 to 3 at these SNR values, results of protocols $\mathrm{LS}_{2}, \mathrm{LP}_{2} \mathrm{~S}_{1}$ and $\mathrm{LP}_{2} \mathrm{~S}_{2}$ are significatively different from those of other protocols (p-values $<0.01)$, while being mostly similar between themself ( $\mathrm{p}$-values $>0.01$ ), according to the p-values calculated from t-tests. These protocols are consistently more precise and also show better accuracy, being closer to the ground truth most of the time in voxels 1 to 3 . As for voxels 4 and 5, all protocols with noisy data produce approximately the same overestimated $\mu \mathrm{FA}$ and are not statistically different (p-values > 0.01). Still for $\mathrm{SNR}=15,20$ and 30, protocols $\mathrm{LP}_{2}, \mathrm{LS}_{1}$ and $\mathrm{LP}_{1} \mathrm{~S}_{1}$ all perform similarly for each voxel (p-values $>0.01$ ), while protocol $\mathrm{LP}_{1}$ has by far the worst accuracy and precision, especially in voxels 1 to 3 . However, it is important to notice that protocol $\mathrm{LP}_{2}$ at $\mathrm{SNR}=20$ is still statistically different then protocols $\mathrm{LS}_{2}$, $\mathrm{LP}_{2} \mathrm{~S}_{1}$ and $\mathrm{LP}_{2} \mathrm{~S}_{2}$ at $\mathrm{SNR}=15$. Furthermore, its precision and accuracy at $\mathrm{SNR}=20$ are also lower then protocols $\mathrm{LS}_{2}, \mathrm{LP}_{2} \mathrm{~S}_{1}$ and $\mathrm{LP}_{2} \mathrm{~S}_{2}$ at $\mathrm{SNR}=15$, even though they are much closer. 
bioRxiv preprint doi: https://doi.org/10.1101/2021.04.07.438845; this version posted May 25, 2021. The copyright holder for this preprint (which was not certified by peer review) is the author/funder, who has granted bioRxiv a license to display the preprint in perpetuity. It is made available under aCC-BY 4.0 International license.

\subsection{In vivo data}

Figures 9 and 11 show memsmt-CSD outputs with protocol $\mathrm{LS}_{2}$ on in vivo data acquired with resolutions of $2.5 \times 2.5 \times 2.5 \mathrm{~mm}^{3}$ and $2 \times 2 \times 2 \mathrm{~mm}^{3}$ respectively, following the preprocessing steps discussed in section 3.7 (see figure A.15 for examples of raw data). Once again, the RGB coded volume fractions allow to distinguish between the three studied tissues, WM, GM and CSF. More precisely, these figures display a zoom of

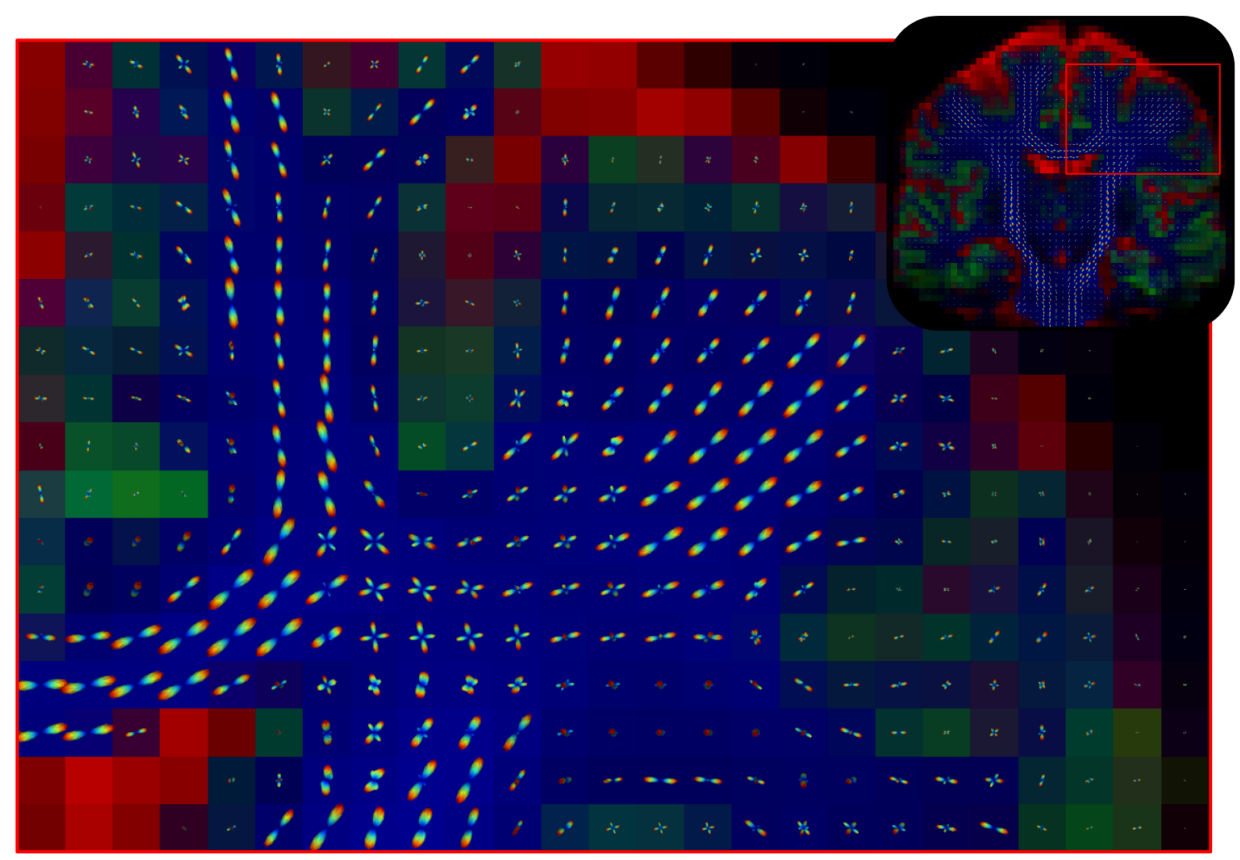

Figure 9: Section of an in vivo human brain coronal slice at $2.5 \mathrm{~mm}$ isotropic showing the WM fODFs obtained from memsmt-CSD with protocol $\mathrm{LS}_{2}$, on top of the computed volume fractions map. The VF is RGB coded, with red being the CSF, green the GM and blue the WM.

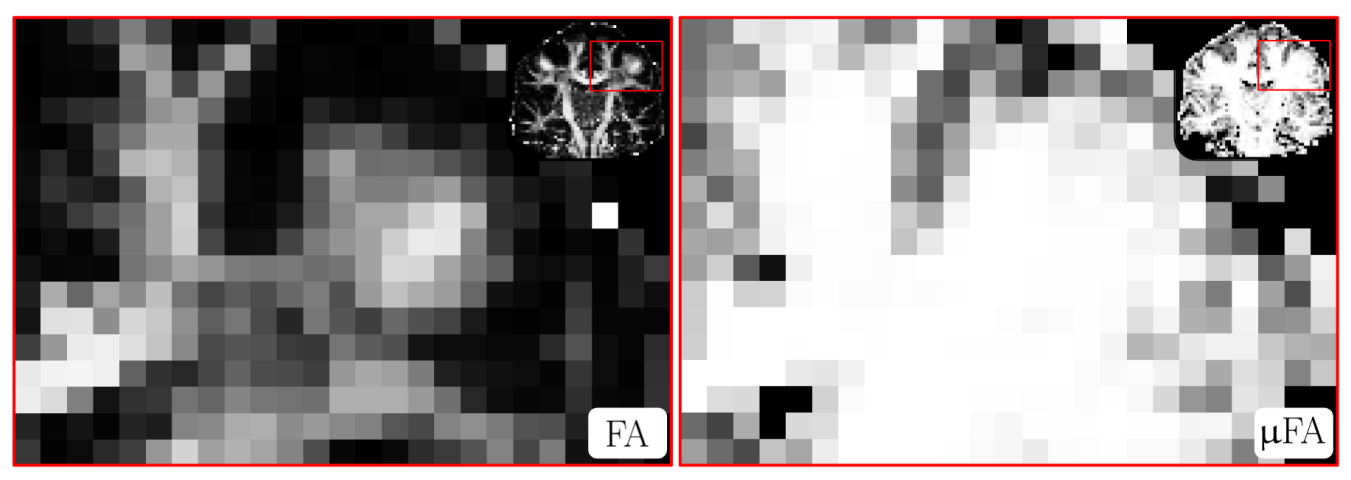

Figure 10: Same brain coronal slice as figure 9, still at $2.5 \mathrm{~mm}$ isotropic, showing the FA computed from DTI with protocol $\mathrm{L}$ and the $\mu \mathrm{FA}$ computed from DIVIDE with protocol $\mathrm{LS}_{2}$. Both measures have values going from 0 (black) to 1 (white). 
bioRxiv preprint doi: https://doi.org/10.1101/2021.04.07.438845; this version posted May 25, 2021. The copyright holder for this preprint (which was not certified by peer review) is the author/funder, who has granted bioRxiv a license to display the preprint in perpetuity. It is made available under aCC-BY 4.0 International license.

chosen brain slice that exhibit voxels similar to the fictional anatomy studied by simulation. Indeed, figures 9 and 11 contain pure single WM fiber and WM fiber crossing voxels, as well as CSF and GM voxels. In the top right of these figures, an image of the whole brain slice helps locating the zoomed image.

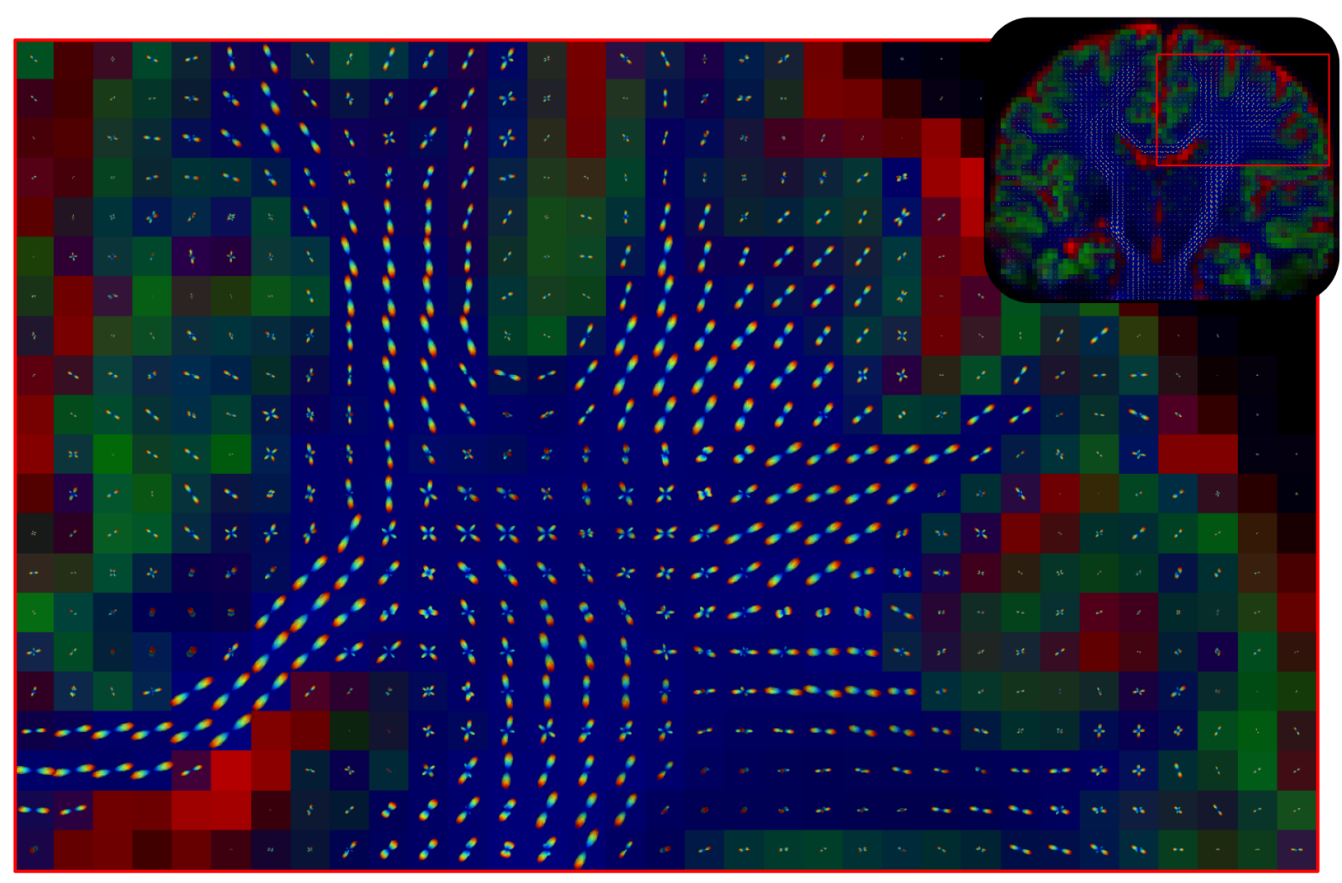

Figure 11: Section of an in vivo human brain coronal slice at $2.0 \mathrm{~mm}$ isotropic showing the WM fODFs obtained from memsmt-CSD with protocol $\mathrm{LS}_{2}$, on top of the computed volume fractions map. The VF is RGB coded, with red being the CSF, green the GM and blue the WM.

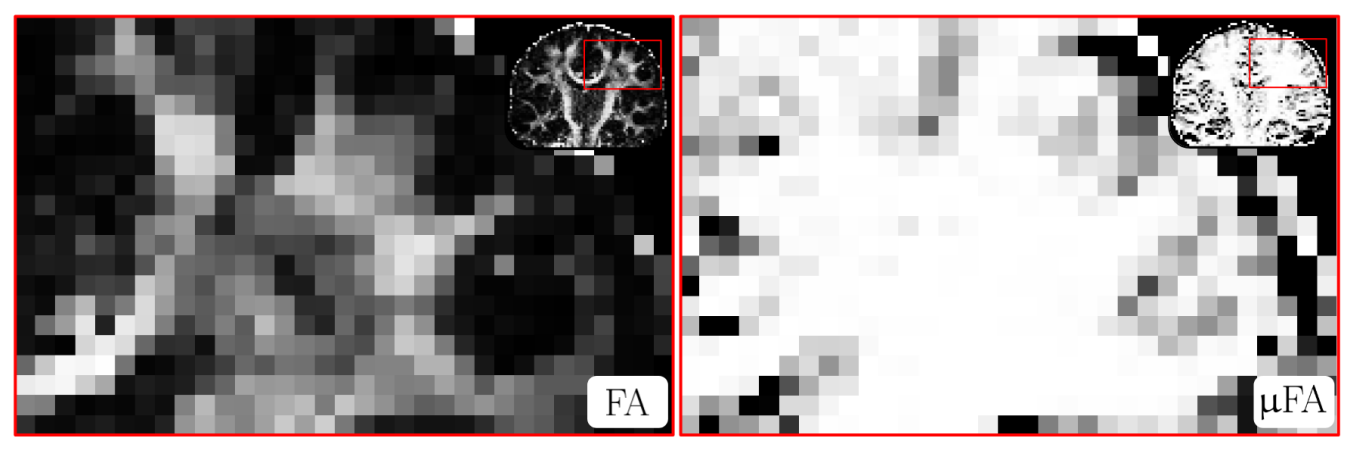

Figure 12: Same brain coronal slice as figure 11, still at $2.0 \mathrm{~mm}$ isotropic, showing the FA computed from DTI with protocol $\mathrm{L}$ and the $\mu \mathrm{FA}$ computed from DIVIDE with protocol $\mathrm{LS}_{2}$. Both measures have values going from 0 (black) to 1 (white).

Figures 10 and 12 present the FA and $\mu \mathrm{FA}$ for the same brain slice as figures 9 and 11, respectively. The FA, calculated only from the LTE protocol (L), shows high intensity 
bioRxiv preprint doi: https://doi.org/10.1101/2021.04.07.438845; this version posted May 25, 2021. The copyright holder for this preprint (which was not certified by peer review) is the author/funder, who has granted bioRxiv a license to display the preprint in perpetuity. It is made available under aCC-BY 4.0 International license.

voxels where single WM fibers are present, and lower intensity where WM fibers are crossing (see figures 9 and 11 for comparison with fODFs). The $\mu \mathrm{FA}$ in the other hand, calculated from the combination of LTE and STE for protocol $\mathrm{LS}_{2}$, shows high intensity voxels for all of the WM voxels, regardless of the presence of crossings.

Figure 13 presents msmt-CSD outputs obtained with the short echo time (86 ms) version of protocol $\mathrm{L}_{\mathrm{msmt}}$ on in vivo data acquired with a resolution of $2 \times 2 \times 2 \mathrm{~mm}^{3}$.

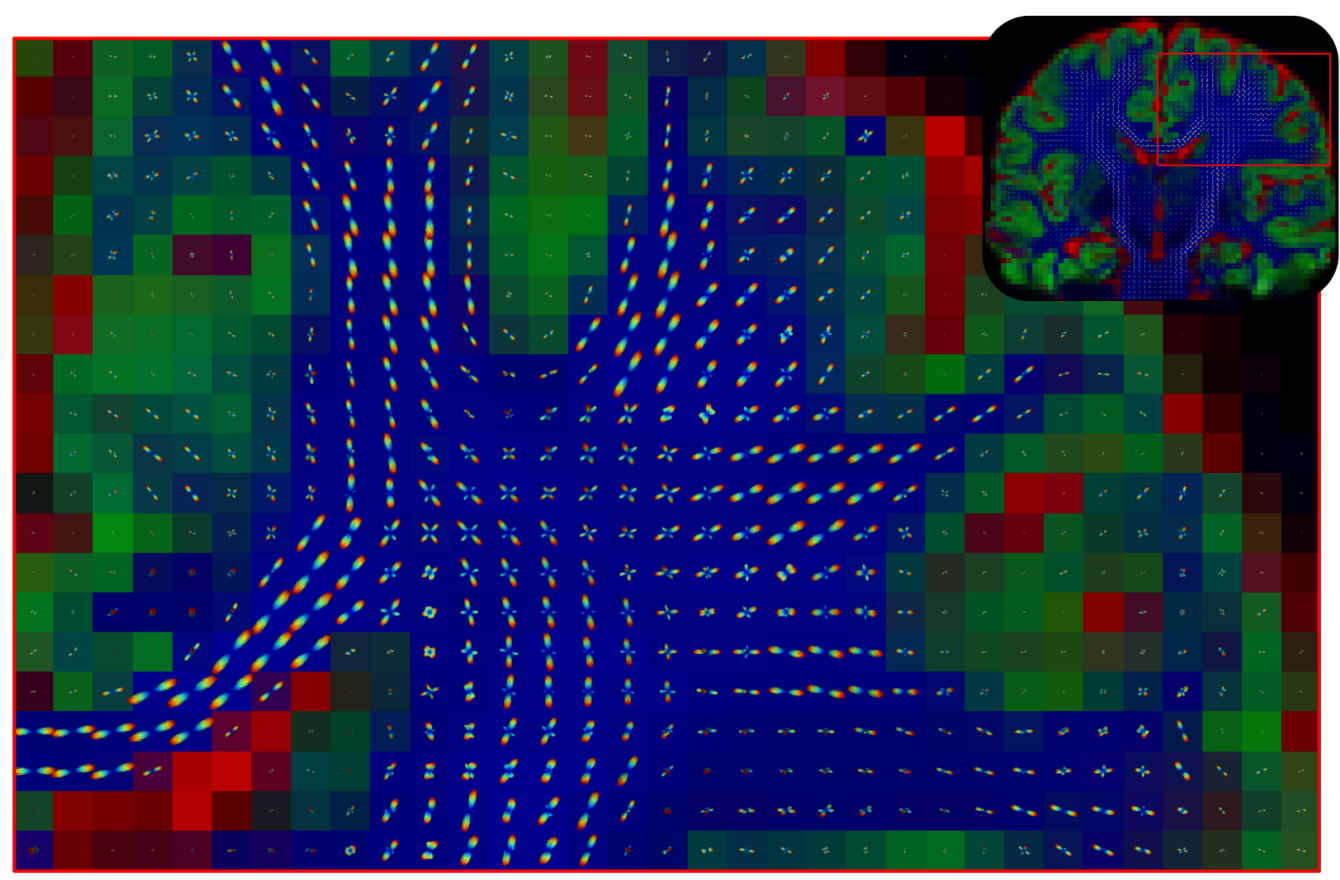

Figure 13: Section of an in vivo human brain coronal slice at $2.0 \mathrm{~mm}$ isotropic showing the WM fODFs obtained from msmt-CSD with the short TE version of protocol $\mathrm{L}_{\mathrm{msmt}}$, on top of the computed volume fractions map. The VF is RGB coded, with red being the CSF, green the GM and blue the WM.

\section{Discussion}

\subsection{Choices of b-values and number of directions per shell}

The choices of b-values and number of gradient directions per shell, previously presented in table 3, were inspired by [Nilsson et al., 2020]. In this paper, the authors present a three-minute tensor-valued dMRI protocol enabling the calculation of the diffusional variances, and thus, the $\mu \mathrm{FA}$, via a powder-averaged two-term cumulant expansion approach. This approach is comparable to the DIVIDE method used in our study, according to Reymbaut et al. [2020c]. The adaptations made from Nilsson et al. [2020] affect both the b-values and the number of directions per shells. Their protocol consisted on four b-values $\left(b=100,700,1400\right.$, and $\left.2000 \mathrm{~s} / \mathrm{mm}^{2}\right)$ acquired in $3,3,6$, and 6 directions for the linear encoding, and 6, 6, 10, and 16 directions for the spherical encoding. We modified the b-values to get a $b=1200 \mathrm{~s} / \mathrm{mm}^{2}$ for the DTI fit [Jones and Basser, 2004], swapping it with the $b=1400 \mathrm{~s} / \mathrm{mm}^{2}$. We also diminished the $b=2000 \mathrm{~s} / \mathrm{mm}^{2}$ to a $b=$ 
$1800 \mathrm{~s} / \mathrm{mm}^{2}$ to keep a smaller gap between the b-values to help fit equation 6 , and we added a $b=2400 \mathrm{~s} / \mathrm{mm}^{2}$ shell for CSD purposes. As shown by figure 8 , this distribution of b-values seems to provide sufficient coverage to get proper $\mu \mathrm{FA}$ values.

The number of directions per shell taken from Nilsson et al. [2020] ensures a good enough directional coverage for the rotation invariant criteria. However, this number is too low to perform CSD, or even to get a well defined DTI fit. Based on the results from Jones [2004], we chose to take 12 directions for the $b=1200 \mathrm{~s} / \mathrm{mm}^{2}$ shell in linear encoding. Figure 3 indeed shows that the FA calculated from DTI with this configuration is in agreement with the literature [Pierpaoli and Basser, 1996], with high values in voxels of single WM fiber (voxel 2), lower values in WM fiber crossings (voxel 1) and near-zero values in voxels with only isotropic contents (voxel 4 and 5). This figure also justifies the choice of 12 directions by the fact that the FA does not change much from data without noise to data of $\mathrm{SNR}=15$. Note that the DTI fit can also be achieved on planar and spherical encodings, allowing the calculation of FA and MD with planar encoding and MD with spherical encoding. However, we chose to use only the linear encoding for DTI measures computation, as fits from other encodings seemed to be heavily sensitive to noise. As for the two higher b-values, 18 and 24 directions were taken, respectively, for a total of 54 directions over $b=1000 \mathrm{~s} / \mathrm{mm}^{2}$ available for the standard msmt-CSD with linear encoding. The performances of protocol $\mathrm{L}$ for $\mathrm{SNR}=15$, presented on figures 6 and 7 , confirm that this amount of directions, combined with the lower shells, is enough to propel the angular resolution up to 55 degrees with msmt-CSD.

Since the proposed number of directions for the spherical encoded gradients $\mathrm{S}_{2}$ mostly serves for SNR purposes and not for directional coverage, as a single spherical encoded signal is already rotation invariant [Szczepankiewicz, 2016], we reduced the number of directions to 3, 3, 6 and 6 , creating a spherical encoding gradient table $\mathrm{S}_{1}$ with shorter acquisition time, at the price of lower SNR. Figure 8 show that for low SNR (15), the DIVIDE method is indeed affected by the decrease of STE directions, as $\mathrm{LS}_{1}$ is significantly less precise than $\mathrm{LS}_{2}$. As for memsmt-CSD, it does not seem to be impacted by this effect, as demonstrated by figure 7 , where both $\mathrm{LS}_{1}$ and $\mathrm{LS}_{2}$ perform as well as the LTE only protocol (L). Therefore, reducing the number of directions from $\mathrm{S}_{2}$ to $\mathrm{S}_{1}$ might not be good for $\mu \mathrm{FA}$ computation, but it does not affect the fODFs computation.

In addition to linear and spherical encodings, two planar encoding gradient tables $\mathrm{P}_{1}$ and $\mathrm{P}_{2}$ were created with the same amount of directions that were tested for linear encoding by Nilsson et al. [2020]. The smaller one, $\mathrm{P}_{1}$, should be sufficient to get rotation invariant signals, since planar encoding requires less directions than linear encoding in that matter [Szczepankiewicz, 2016; Szczepankiewicz et al., 2019]. Considering this, the results from figure 8 , that show that protocol $\mathrm{LP}_{1}$ is the worst in terms of accuracy and precision when it comes to $\mu \mathrm{FA}$ computation with noise, even when comparing at $\mathrm{SNR}=20$ to take into account a possible lower TE due to the absence of STE, probably indicate that planar tensor encoding suffers more from noise than spherical tensor encoding in terms of DIVIDE. Indeed, even with more directions (LP2) at SNR=20, PTE still does not produce results as good as protocol $\mathrm{LS}_{2}$ at $\mathrm{SNR}=15$, which has the same amount of directions.

\subsection{Effects of protocol choice on the computation of $\mu F A$}

The choice of protocol has a big impact on the accuracy and the precision of the computed $\mu \mathrm{FA}$, as shown by figure 8 . According to this figure, PTE alone with LTE 
$\left(\mathrm{LP}_{1}\right.$ and $\left.\mathrm{LP}_{2}\right)$ is consistently underperforming other protocols in voxels containing WM (voxels 1 to 3 ) and when comparing at equal SNR. In these voxels, we demonstrate that STE and LTE combined can produce accurate and precise measures of $\mu F A$, if a sufficient amount of directions is used, which is the case of $\mathrm{LS}_{2}$. This observation agrees with the conclusions of Nilsson et al. [2020] about their proposed protocol, very similar to $\mathrm{LS}_{2}$. Furthermore, the use of three tensor shapes with enough directions can also provide good $\mu \mathrm{FA}$ measures, as $\mathrm{LP}_{2} \mathrm{~S}_{1}$ and $\mathrm{LP}_{2} \mathrm{~S}_{2}$ display accuracy and precision very similar to $\mathrm{LS}_{2}$. However, the higher acquisition times needed for $\mathrm{LP}_{2} \mathrm{~S}_{1}$ and $\mathrm{LP}_{2} \mathrm{~S}_{2}$ do not procure significant advantage to the computation of the $\mu \mathrm{FA}$, leaving $\mathrm{LS}_{2}$ with the best time to quality ratio. Moreover, when taking into account the fact that STE requires higher TE then LTE or PTE and thus suffers from lower SNR values, the use of a protocol such as $\mathrm{LP}_{2}$ becomes interesting. Nevertheless, figure 8 demonstrates that even when comparing $\mathrm{LP}_{2}$ at $\mathrm{SNR}=20$ with $\mathrm{LS}_{2}$ at $\mathrm{SNR}=15$, which mimics the effect of various echo times similar to the ones used in the in vivo acquisitions, the $\mathrm{LS}_{2}$ protocol is still producing slightly better results then $\mathrm{LP}_{2}$.

In voxels 4 and 5, composed of GM and CSF respectively, a constant overestimation of the $\mu \mathrm{FA}$ is observed. This is due to the noise inducing apparent anisotropy at the microscopic scales, increasing the $\mu \mathrm{FA}$. Indeed, this is confirmed by the fact that this effect appears only when adding noise, since $\mu \mathrm{FA}$ is accurately estimated at $\mathrm{SNR}=\infty$ and starts to deviate more and more with added noise. In fact, this increase of anisotropy measures with noise is a well known phenomenon discussed in Pierpaoli and Basser [1996]; Jones and Basser [2004] and caused by eigenvalue repulsion [Mehta, 2004]. Ultimately, no protocol stands out as the better one for these two voxels.

\subsection{Effects of protocol choice on the angular resolution of a WM fiber crossing}

The choice of protocol has a smaller impact for the results of memsmt-CSD than it has for the DIVIDE method. Indeed, figures 4, 5 and 6 present the effects of adding planar and/or spherical tensor shapes to a LTE protocol. Instinctively, it would be surprising to see a complete breakdown when adding additional tensor-valued encodings at a constant SNR level. As a matter of fact, figure 7 shows that adding a STE gradient table $\left(\mathrm{S}_{1}\right.$ or $\mathrm{S}_{2}$ ) to the linear $(\mathrm{L})$ protocol does not diminish the minimal angle at which CSD is able to distinguish two WM fibers crossing. Nevertheless, the figure also shows that adding either of the two PTE gradient tables $\left(\mathrm{P}_{1}\right.$ or $\left.\mathrm{P}_{2}\right)$ to the linear $(\mathrm{L})$ protocol decreases the angular resolution of the CSD method by 3 degrees at $\mathrm{SNR}=15$ and by 1 degree at $\mathrm{SNR}=20$. This effect is indeed more visible at low SNR, meaning that PTE might not react well with noise when it comes to distinguishing two WM fiber populations. Adding STE to $\mathrm{LP}_{1}$ or $\mathrm{LP}_{2}$ seems to lower this negative effect, as it allows memsmt$\mathrm{CSD}$ to reach 1 more degree of separation at $\mathrm{SNR}=15$. Once again, to provide with a fairer comparison, protocols containing STE should be compared at SNR=15 with other protocols at $\mathrm{SNR}=20$, to emulate the effect of a shorter TE. When doing so, it appears that protocols $\mathrm{LS}_{1}$ and $\mathrm{LS}_{2}$ indeed have the same angular resolution as protocols $\mathrm{LP}_{1}$ and $\mathrm{LP}_{2}$ (55 degrees) and that these protocols all have 1 less degree of angular resolution then protocol L. Furthermore, it is worth noting that the typical multi-shell protocol with the same amount of gradient directions as protocols $\mathrm{LS}_{2}$ or $\mathrm{LP}_{2}$ has 3 degrees more of angular resolution at $\mathrm{SNR}=20$. However, protocol $\mathrm{L}_{\mathrm{msmt}}$ does not provide $\mu \mathrm{FA}$ measures with DIVIDE. If the acquisition time is brought to the discussion (see table 5), three tensor shapes protocols $\left(\mathrm{LP}_{1} \mathrm{~S}_{1}, \mathrm{LP}_{2} \mathrm{~S}_{1}\right.$ and $\left.\mathrm{LP}_{2} \mathrm{~S}_{2}\right)$ are not worth the longer time, and 
protocols $\mathrm{LS}_{1}, \mathrm{LS}_{2}, \mathrm{LP}_{1}$ and $\mathrm{LP}_{2}$ all stand out as the better choices of multi-encoding protocols for computing WM fibers crossing with memsmt-CSD.

\subsection{Combined memsmt-CSD and DIVIDE performances}

While figure 3 serves as a proof of concept for the memsmt-CSD, DTI fit and DIVIDE methods, figures 7 and 8 bring to light the importance of the chosen protocol for fODFs angular resolution and $\mu \mathrm{FA}$ computation. Indeed, when combining the conclusions of section 5.2 and 5.3, protocol $\mathrm{LS}_{2}$ arises as the best choice for computing both memsmtCSD and DIVIDE. Even if the typical $\mathrm{L}_{\mathrm{msmt}}$ protocol shows the better results for CSD, reorganising the gradient directions distribution to create $\mathrm{LS}_{2}$ from the same amount of directions only leads to the lost of a few degrees of angular resolution, while enabling the DIVIDE process. Still, protocol $\mathrm{LP}_{2}$ must not be forgotten, as it has proven to be comparable to $\mathrm{LS}_{2}$ in terms of angular resolution with memsmt-CSD and to produce $\mu \mathrm{FA}$ measures that are only slightly less precise and accurate then protocol $\mathrm{LS}_{2}$.

Figures 9 and 10 provide an example of what protocol $\mathrm{LS}_{2}$ can achieve in terms of WM fODF reconstruction and measures calculation at a typical resolution for tensorvalued dMRI data (2.5 mm isotropic). Figures 11 and 12 show that this resolution can be increased to $2.0 \mathrm{~mm}$ isotropic and still produce valuable WM fODF reconstruction and measures calculation. Figures 9 and 11 show that memsmt-CSD, combined with protocol $\mathrm{LS}_{2}$, is able to produce clean WM fODFs in WM voxels, but most importantly in crossing fibers voxels. Furthermore, it is able to distinguish WM from GM and CSF, rendering a sort of tissue segmentation. Figures 10 and 12 show that the LTE part of protocol $\mathrm{LS}_{2}$ can be used to produce a FA map that represent very well the WM voxel, when compared to the WM segmentation from memsmt-CSD, apart from the famous FA drop in crossing fibers voxels. Besides, figures 10 and 12 also show that the same protocol can be used to compute a $\mu \mathrm{FA}$ map, which does not suffer like the FA in crossing fibers. However, the $\mu \mathrm{FA}$ seems to be swelled a bit, especially in regions of WM and GM interface. This phenomena is probably attributable to the noise level of these in vivo acquisition, but perhaps a better calibration of the DIVIDE method could be done. Nevertheless, those figures prove that memsmt-CSD is viable and that fODFs, FA and $\mu \mathrm{FA}$ can all be computed from protocol $\mathrm{LS}_{2}$.

Moreover, figure 13 allows to compare the performances of msmt-CSD and memsmtCSD, using protocol $\mathrm{L}_{\mathrm{msmt}}$ with a lower echo time. It appears that both methods produce similar fODFs and volume fractions map separating the different tissues. However, it is also clear that msmt-CSD and memsmt-CSD do not reconstruct the same results. Indeed, the GM parts of the VF map seem to be different and the fODFs in WM crossing also show differences.

\subsection{Recommendations and future work}

Considering all of the above, we recommend using our combination of LTE and STE gradient tables to create the $\mathrm{LS}_{2}$ protocol in the scope of computing both fODFs and $\mu \mathrm{FA}$. Indeed, this protocol uses two different tensor encoding shapes for an accurate and precise computation of the $\mu \mathrm{FA}$ with a DIVIDE method, while not losing too much angular resolution with memsmt-CSD in comparison to similar msmt-CSD. Furthermore, its acquisition time of approximately 10 minutes at a resolution of $2.5 \mathrm{~mm}$ isotropic makes it attractive for research purposes. If possible, we recommend spending 3 more 
minutes to push the resolution up to $2.0 \mathrm{~mm}$ isotropic and get less partial volume voxels. As structural connectivity, tractometry and connectomics is becoming more and more present in research and disease applications, it is important to push for higher spatial resolution multi-dimensional acquisition in clinically feasible times.

While the in vivo results of memsmt-CSD are promising, the computed $\mu \mathrm{FA}$ could benefit from more denoising or better DIVIDE tuning. Nevertheless, the proposed protocol is a starting point for future research, where fODFs and $\mu \mathrm{FA}$ are both of interest. Further investigation of the impact of multiple b-tensor shapes on the VF values and fODF reconstruction in voxels of partial volume would be needed. Indeed, the interesting problem of the presence of an isotropic compartment inside a WM fiber crossing voxel could be better suited for a protocol containing STE, like $\mathrm{LS}_{2}$. Moreover, the impact of planar tensor encoding on memsmt-CSD should be explored in more depth and is not to be forgotten. Further investigation of the pros and cons of memsmt-CSD, especially with STE, could provide a better understanding of the effects of this technique on microstructure estimation at the voxel level but also at the tractography and connectome level. More digging and a better comparison with msmt-CSD should be done, especially regarding the difference in the $\mathrm{VF}$ maps.

\section{Conclusion}

In this work, we first established the mathematical and computational foundations of a multi-encoding msmt-CSD model, able to compute fODFs and volume fraction maps from tensor-valued dMRI data. Using simulated data, we showed that the model can indeed produce multi-tissue volume fraction maps and white matter fODFs in single and crossing fibers voxels. Furthermore, these fODFs only suffer from the lost of a few degrees in terms of angular resolution when adding sufficient amount of spherical tensor encoding or planar tensor encoding acquisitions to a linear tensor encoding. Moreover, the performance of different combinations of linear, planar and spherical gradient tables were also evaluated with the DIVIDE method on the simulated data. We showed that while combining three b-tensor shapes provides great accuracy and precision in $\mu \mathrm{FA}$ computation, combining only linear and spherical b-tensors also yields competing accuracy and precision values, while producing satisfying fODF reconstruction with memst-CSD.

We thus propose a $10 \mathrm{~min}$ protocol at $2.5 \mathrm{~mm}$ isotropic and a $13 \mathrm{~min}$ protocol at 2 $\mathrm{mm}$ isotropic combining linear and spherical b-tensor encodings to get both memsmtCSD and diffusional variance decomposition methods. These protocols therefore kill two birds with one stone, allowing the reconstruction of accurate crossing fiber fODFs for tractography/connectivity while being able to extract the $\mu \mathrm{FA}$ map precisely.

\section{Acknowledgements}

The authors wish to thank François Rheault and Guillaume Theaud for their help with the implementation of the memsmt-CSD method in DIPY, as well as Charles Poirier for his help with fODF visualization with variances. They are also grateful to the Fonds de recherche du Québec - Nature et technologies (FRQNT) and the Natural Sciences and Engineering Research Council of Canada (NSERC) programs for funding this research. The authors also thank Prof. Christine Tardif and Prof. Pascal Tétreault for their advice on this paper. 
They also wish to thank the HCP project for the use of their data for estimating the non-DW signals per tissues. The HCP project (Principal Investigators : Bruce Rosen, M.D., Ph.D., Martinos Center at Massachusetts General Hospital; Arthur W. Toga, Ph.D., University of Southern California, Van J. Weeden, MD, Martinos Center at Massachusetts General Hospital) is supported by the National Institute of Dental and Craniofacial Research (NIDCR), the National Institute of Mental Health (NIMH) and the National Institute of Neurological Disorders and Stroke (NINDS). Collectively, the HCP is the result of efforts of co-investigators from the University of Southern California, Martinos Center for Biomedical Imaging at Massachusetts General Hospital (MGH), Washington University, and the University of Minnesota.

\section{References}

Agrawal, A., Verschueren, R., Diamond, S., Boyd, S., 2018. A rewriting system for convex optimization problems. Journal of Control and Decision 5, 42-60.

Alexander, D.C., 2008. A general framework for experiment design in diffusion mri and its application in measuring direct tissue-microstructure features. Magnetic Resonance in Medicine: An Official Journal of the International Society for Magnetic Resonance in Medicine 60, 439-448.

Alexander, D.C., Hubbard, P.L., Hall, M.G., Moore, E.A., Ptito, M., Parker, G.J., Dyrby, T.B., 2010. Orientationally invariant indices of axon diameter and density from diffusion mri. Neuroimage 52, 1374-1389.

de Almeida Martins, J.P., Tax, C.M., Reymbaut, A., Szczepankiewicz, F., Chamberland, M., Jones, D.K., Topgaard, D., 2021. Computing and visualising intra-voxel orientation-specific relaxationdiffusion features in the human brain. Human brain mapping 42, 310-328.

Andersen, K.W., Lasič, S., Lundell, H., Nilsson, M., Topgaard, D., Sellebjerg, F., Szczepankiewicz, F., Siebner, H.R., Blinkenberg, M., Dyrby, T.B., 2020. Disentangling white-matter damage from physiological fibre orientation dispersion in multiple sclerosis. Brain communications 2, fcaa077.

Andersson, J.L., Skare, S., Ashburner, J., 2003. How to correct susceptibility distortions in spin-echo echo-planar images: application to diffusion tensor imaging. Neuroimage 20, 870-888.

Avants, B.B., Tustison, N., Song, G., 2009. Advanced normalization tools (ants). Insight j 2, 1-35.

Basser, P.J., Mattiello, J., LeBihan, D., 1994. Mr diffusion tensor spectroscopy and imaging. Biophysical journal 66, 259-267.

Basser, P.J., Pajevic, S., 2003. A normal distribution for tensor-valued random variables: applications to diffusion tensor mri. IEEE Transactions on Medical Imaging 22, 785-794.

Basser, P.J., Pierpaoli, C., 1996. Microstructural and physiological features of tissues elucidated by quantitative-diffusion-tensor mri. Journal of Magnetic Resonance, Series B 111, 209-219.

Caruyer, E., Lenglet, C., Sapiro, G., Deriche, R., 2013. Design of multishell sampling schemes with uniform coverage in diffusion mri. Magnetic resonance in medicine 69, 1534-1540.

Catani, M., Howard, R.J., Pajevic, S., Jones, D.K., 2002. Virtual in vivo interactive dissection of white matter fasciculi in the human brain. NeuroImage 17, 77-94.

Conturo, T.E., McKinstry, R.C., Akbudak, E., Robinson, B.H., 1996. Encoding of anisotropic diffusion with tetrahedral gradients: a general mathematical diffusion formalism and experimental results. Magnetic Resonance in Medicine 35, 399-412.

Cottaar, M., Szczepankiewicz, F., Bastiani, M., Hernandez-Fernandez, M., Sotiropoulos, S.N., Nilsson, M., Jbabdi, S., 2020. Improved fibre dispersion estimation using b-tensor encoding. NeuroImage 215, 116832.

Coupé, P., Yger, P., Prima, S., Hellier, P., Kervrann, C., Barillot, C., 2008. An optimized blockwise nonlocal means denoising filter for 3-d magnetic resonance images. IEEE transactions on medical imaging 27, 425-441.

Descoteaux, M., 2008. High angular resolution diffusion MRI: from local estimation to segmentation and tractography. Ph.D. thesis. INRIA Sophia Antipolis. France.

Descoteaux, M., Angelino, E., Fitzgibbons, S., Deriche, R., 2007. Regularized, fast, and robust analytical q-ball imaging. Magnetic Resonance in Medicine 58, 497-510.

Descoteaux, M., Deriche, R., Knosche, T.R., Anwander, A., 2009. Deterministic and probabilistic tractography based on complex fibre orientation distributions. IEEE Transactions on Medical Imaging $28,269-286$. 
Diamond, S., Boyd, S., 2016. CVXPY: A Python-embedded modeling language for convex optimization. Journal of Machine Learning Research 17, 1-5.

Douaud, G., Jbabdi, S., Behrens, T.E., Menke, R.A., Gass, A., Monsch, A.U., Rao, A., Whitcher, B. Kindlmann, G., Matthews, P.M., et al., 2011. Dti measures in crossing-fibre areas: increased diffusion anisotropy reveals early white matter alteration in mci and mild alzheimer's disease. Neuroimage 55, $880-890$.

Eriksson, S., Lasič, S., Nilsson, M., Westin, C.F., Topgaard, D., 2015. Nmr diffusion-encoding with axial symmetry and variable anisotropy: Distinguishing between prolate and oblate microscopic diffusion tensors with unknown orientation distribution. The Journal of chemical physics 142, 104201.

Eriksson, S., Lasič, S., Topgaard, D., 2013. Isotropic diffusion weighting in pgse nmr by magic-angle spinning of the q-vector. Journal of Magnetic Resonance 226, 13-18.

Garyfallidis, E., Brett, M., Amirbekian, B., Rokem, A., Van Der Walt, S., Descoteaux, M., NimmoSmith, I., 2014. Dipy, a library for the analysis of diffusion mri data. Frontiers in neuroinformatics 8, 8.

Glasser, M.F., Sotiropoulos, S.N., Wilson, J.A., Coalson, T.S., Fischl, B., Andersson, J.L., Xu, J., Jbabdi, S., Webster, M., Polimeni, J.R., et al., 2013. The minimal preprocessing pipelines for the human connectome project. Neuroimage 80, 105-124.

Jensen, J.H., Helpern, J.A., Ramani, A., Lu, H., Kaczynski, K., 2005. Diffusional kurtosis imaging: the quantification of non-gaussian water diffusion by means of magnetic resonance imaging. Magnetic Resonance in Medicine: An Official Journal of the International Society for Magnetic Resonance in Medicine 53, 1432-1440.

Jeurissen, B., Leemans, A., Tournier, J.D., Jones, D.K., Sijbers, J., 2013. Investigating the prevalence of complex fiber configurations in white matter tissue with diffusion magnetic resonance imaging. Human Brain Mapping 34, 2747-2766.

Jeurissen, B., Szczepankiewicz, F., June 2018. Spherical deconvolution of diffusion mri data with tensorvalued encodings, in: Proceedings of the joint annual meeting ISMRM-ESMRMB, Paris, France. p. 1559.

Jeurissen, B., Tournier, J.D., Dhollander, T., Connelly, A., Sijbers, J., 2014. Multi-tissue constrained spherical deconvolution for improved analysis of multi-shell diffusion mri data. NeuroImage 103, $411-426$.

Jian, B., Vemuri, B.C., Özarslan, E., Carney, P.R., Mareci, T.H., 2007. A novel tensor distribution model for the diffusion-weighted mr signal. NeuroImage 37, 164-176.

Jones, D.K., 2004. The effect of gradient sampling schemes on measures derived from diffusion tensor mri: a monte carlo study. Magnetic Resonance in Medicine: An Official Journal of the International Society for Magnetic Resonance in Medicine 51, 807-815.

Jones, D.K., Basser, P.J., 2004. "squashing peanuts and smashing pumpkins": how noise distorts diffusion-weighted mr data. Magnetic Resonance in Medicine: An Official Journal of the International Society for Magnetic Resonance in Medicine 52, 979-993.

Jones, D.K., Cercignani, M., 2010. Twenty-five pitfalls in the analysis of diffusion mri data. NMR in Biomedicine 23, 803-820.

Kamiya, K., Kamagata, K., Ogaki, K., Hatano, T., Ogawa, T., Takeshige-Amano, H., Murata, S. Andica, C., Murata, K., Feiweier, T., et al., 2020. Brain white-matter degeneration due to aging and parkinson disease as revealed by double diffusion encoding. Frontiers in Neuroscience 14, 1091.

Lasič, S., Szczepankiewicz, F., Eriksson, S., Nilsson, M., Topgaard, D., 2014. Microanisotropy imaging: quantification of microscopic diffusion anisotropy and orientational order parameter by diffusion mri with magic-angle spinning of the q-vector. Frontiers in Physics 2, 11.

Le Bihan, D., Breton, E., 1985. Imagerie de diffusion in vivo par résonance magnétique nucléaire. Comptes rendus de l'Académie des sciences. Série 2, Mécanique, Physique, Chimie, Sciences de l'univers, Sciences de la Terre 301, 1109-1112.

Liu, T., Young, G., Huang, L., Chen, N.K., Wong, S.T., 2006. 76-space analysis of grey matter diffusivity: methods and applications. NeuroImage 31, 51-65.

Lundell, H., Lasič, S., 2020. Chapter 2 diffusion encoding with general gradient waveforms, in: Advanced Diffusion Encoding Methods in MRI. The Royal Society of Chemistry, pp. 12-67.

Mehta, M.L., 2004. Random matrices. Elsevier.

Mori, S., Crain, B.J., Chacko, V.P., Van Zijl, P.C.M., 1999. Three-dimensional tracking of axonal projections in the brain by magnetic resonance imaging. Annals of Neurology 45, 265-269.

Naranjo, I.D., Reymbaut, A., Brynolfsson, P., Lo Gullo, R., Bryskhe, K., Topgaard, D., Giri, D.D., Reiner, J.S., Thakur, S.B., Pinker-Domenig, K., 2021. Multidimensional diffusion magnetic resonance imaging for characterization of tissue microstructure in breast cancer patients: A prospective pilot 
study. Cancers 13

Nilsson, M., Szczepankiewicz, F., Brabec, J., Taylor, M., Westin, C.F., Golby, A., van Westen, D., Sundgren, P.C., 2020. Tensor-valued diffusion mri in under 3 minutes: an initial survey of microscopic anisotropy and tissue heterogeneity in intracranial tumors. Magnetic resonance in medicine 83, 608620 .

Nilsson, M., Szczepankiewicz, F., Lampinen, B., Ahlgren, A., de Almeida Martins, J.P., Lasic, S., Westin, C.F., Topgaard, D., 2018. An open-source framework for analysis of multidimensional diffusion mri data implemented in matlab, in: Proc Intl Soc Mag Reson Med, p. 5355.

Pierpaoli, C., Basser, P.J., 1996. Toward a quantitative assessment of diffusion anisotropy. Magnetic resonance in Medicine 36, 893-906.

Poupon, C., Clark, C., Frouin, V., Régis, J., Bloch, I., Le Bihan, D., Mangin, J.F., 2000. Regularization of diffusion-based direction maps for the tracking of brain white matter fascicles. NeuroImage 12, $184-195$.

Reymbaut, A., 2020. Chapter 3 diffusion anisotropy and tensor-valued encoding, in: Advanced Diffusion Encoding Methods in MRI. The Royal Society of Chemistry, pp. 68-102.

Reymbaut, A., Caron, A.V., Gilbert, G., Szczepankiewicz, F., Nilsson, M., Warfield, S., Descoteaux, M., Scherrer, B., 2020a. Magic diamond: Multi-fascicle diffusion compartment imaging with tensor distribution modeling and tensor-valued diffusion encoding. arXiv preprint arXiv:2004.07340 .

Reymbaut, A., Critchley, J., Durighel, G., Sprenger, T., Sughrue, M., Bryskhe, K., Topgaard, D., 2021. Toward nonparametric diffusion-characterization of crossing fibers in the human brain. Magnetic Resonance in Medicine 85, 2815-2827.

Reymbaut, A., Martins, J., Tax, C., Szczepankiewicz, F., Jones, D., Topgaard, D., 2020b. Resolving orientation-specific diffusion-relaxation features via monte-carlo density-peak clustering in heterogeneous brain tissue. arXiv preprint arXiv:2004.08626 .

Reymbaut, A., Mezzani, P., de Almeida Martins, J.P., Topgaard, D., 2020c. Accuracy and precision of statistical descriptors obtained from multidimensional diffusion signal inversion algorithms. NMR in Biomedicine 33, e4267.

Röding, M., Bernin, D., Jonasson, J., Särkkä, A., Topgaard, D., Rudemo, M., Nydén, M., 2012. The gamma distribution model for pulsed-field gradient nmr studies of molecular-weight distributions of polymers. Journal of magnetic resonance 222, 105-111.

Sjölund, J., Szczepankiewicz, F., Nilsson, M., Topgaard, D., Westin, C.F., Knutsson, H., 2015. Constrained optimization of gradient waveforms for generalized diffusion encoding. Journal of Magnetic Resonance 261, 157-168.

Smith, S.M., 2002. Fast robust automated brain extraction. Human brain mapping 17, $143-155$.

Smith, S.M., Jenkinson, M., Woolrich, M.W., Beckmann, C.F., Behrens, T.E., Johansen-Berg, H., Bannister, P.R., De Luca, M., Drobnjak, I., Flitney, D.E., et al., 2004. Advances in functional and structural $\mathrm{mr}$ image analysis and implementation as fsl. Neuroimage 23, S208-S219.

Sotiropoulos, S.N., Jbabdi, S., Xu, J., Andersson, J.L., Moeller, S., Auerbach, E.J., Glasser, M.F., Hernandez, M., Sapiro, G., Jenkinson, M., et al., 2013. Advances in diffusion mri acquisition and processing in the human connectome project. Neuroimage 80, 125-143.

Stellato, B., Banjac, G., Goulart, P., Bemporad, A., Boyd, S., 2020. OSQP: an operator splitting solver for quadratic programs. Mathematical Programming Computation 12, 637-672.

Szczepankiewicz, F., 2016. Imaging diffusional variance by MRI [public]: The role of tensor-valued diffusion encoding and tissue heterogeneity. Ph.D. thesis. Lund University.

Szczepankiewicz, F., Lasič, S., van Westen, D., Sundgren, P.C., Englund, E., Westin, C.F., Ståhlberg, F., Lätt, J., Topgaard, D., Nilsson, M., 2015. Quantification of microscopic diffusion anisotropy disentangles effects of orientation dispersion from microstructure: applications in healthy volunteers and in brain tumors. NeuroImage 104, 241-252.

Szczepankiewicz, F., Sjölund, J., Ståhlberg, F., Lätt, J., Nilsson, M., 2019. Tensor-valued diffusion encoding for diffusional variance decomposition (divide): Technical feasibility in clinical mri systems. PLoS One 14, e0214238.

Szczepankiewicz, F., van Westen, D., Englund, E., Westin, C.F., Ståhlberg, F., Lätt, J., Sundgren, P.C., Nilsson, M., 2016. The link between diffusion mri and tumor heterogeneity: Mapping cell eccentricity and density by diffusional variance decomposition (divide). Neuroimage 142, 522-532.

Szczepankiewicz, F., Westin, C.F., Nilsson, M., 2020. Gradient waveform design for tensor-valued encoding in diffusion mri. Journal of Neuroscience Methods , 109007.

Teipel, S.J., Grothe, M.J., Filippi, M., Fellgiebel, A., Dyrba, M., Frisoni, G.B., Meindl, T., Bokde, A.L. Hampel, H., Klöppel, S., et al., 2014. Fractional anisotropy changes in alzheimer's disease depend on the underlying fiber tract architecture: a multiparametric dti study using joint independent component 
analysis. Journal of Alzheimer's Disease 41, 69-83.

Theaud, G., Houde, J.C., Boré, A., Rheault, F., Morency, F., Descoteaux, M., 2020. Tractoflow: A robust, efficient and reproducible diffusion mri pipeline leveraging nextflow \& singularity. NeuroImage 218,116889 .

Tournier, J.D., Calamante, F., Connelly, A., 2007. Robust determination of the fibre orientation distribution in diffusion mri: non-negativity constrained super-resolved spherical deconvolution. Neuroimage $35,1459-1472$.

Tournier, J.D., Calamante, F., Gadian, D.G., Connelly, A., 2004. Direct estimation of the fiber orientation density function from diffusion-weighted mri data using spherical deconvolution. Neuroimage 23, $1176-1185$

Tuch, D.S., Reese, T.G., Wiegell, M.R., Makris, N., Belliveau, J.W., Wedeen, V.J., 2002. High angular resolution diffusion imaging reveals intravoxel white matter fiber heterogeneity. Magnetic Resonance in Medicine 48, 577-582.

Veraart, J., Fieremans, E., Novikov, D.S., 2016. Diffusion mri noise mapping using random matrix theory. Magnetic resonance in medicine 76, 1582-1593.

Volz, L.J., Cieslak, M., Grafton, S., 2018. A probabilistic atlas of fiber crossings for variability reduction of anisotropy measures. Brain Structure and Function 223, 635-651.

Westin, C.F., Knutsson, H., Pasternak, O., Szczepankiewicz, F., Özarslan, E., van Westen, D., Mattisson, C., Bogren, M., O'Donnell, L.J., Kubicki, M., et al., 2016. Q-space trajectory imaging for multidimensional diffusion mri of the human brain. Neuroimage 135, 345-362.

Westin, C.F., Szczepankiewicz, F., Pasternak, O., Özarslan, E., Topgaard, D., Knutsson, H., Nilsson, M., 2014. Measurement tensors in diffusion mri: generalizing the concept of diffusion encoding, in: International conference on medical image computing and computer-assisted intervention, Springer. pp. 209-216.

Wheeler-Kingshott, C.A., Cercignani, M., 2009. About "axial" and "radial" diffusivities. Magnetic Resonance in Medicine: An Official Journal of the International Society for Magnetic Resonance in Medicine 61, 1255-1260.

Zhang, H., Schneider, T., Wheeler-Kingshott, C.A., Alexander, D.C., 2012. Noddi: practical in vivo neurite orientation dispersion and density imaging of the human brain. Neuroimage 61, 1000-1016.

Zhang, Y., Brady, M., Smith, S., 2001. Segmentation of brain mr images through a hidden markov random field model and the expectation-maximization algorithm. IEEE transactions on medical imaging 20, 45-57. 
bioRxiv preprint doi: https://doi.org/10.1101/2021.04.07.438845; this version posted May 25, 2021. The copyright holder for this preprint (which was not certified by peer review) is the author/funder, who has granted bioRxiv a license to display the preprint in perpetuity. It is made available under aCC-BY 4.0 International license.

\section{Appendix A. Datasets \& Databases}


bioRxiv preprint doi: https://doi.org/10.1101/2021.04.07.438845; this version posted May 25, 2021. The copyright holder for this preprint (which was not certified by peer review) is the author/funder, who has granted bioRxiv a license to display the preprint in perpetuity. It is made available under aCC-BY 4.0 International license.

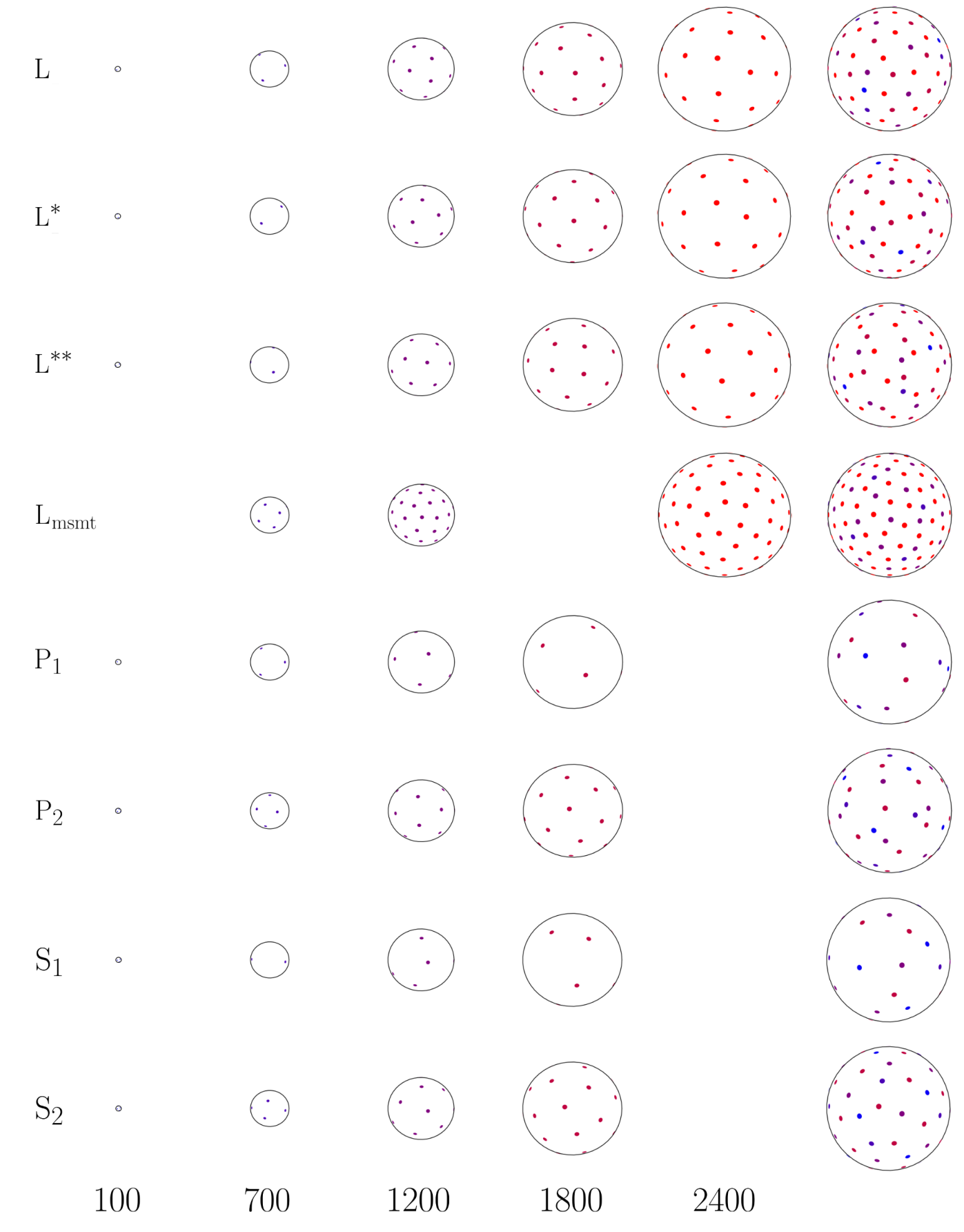

Figure A.14: Visualization of the gradient directions distribution for each protocols studied. The first five columns represent the distribution at b-values of $100,700,1200,1800$ and $2400 \mathrm{~s} / \mathrm{mm}^{2}$. For these columns, the amplitudes of the spheres are proportional to the b-values. The last column is every shell combined on one sphere. The exact gradient tables are available at https://doi.org/10.5281/zenodo.4628539. 
bioRxiv preprint doi: https://doi.org/10.1101/2021.04.07.438845; this version posted May 25, 2021. The copyright holder for this preprint (which was not certified by peer review) is the author/funder, who has granted bioRxiv a license to display the preprint in perpetuity. It is made available under aCC-BY 4.0 International license.
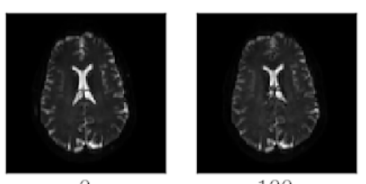

100

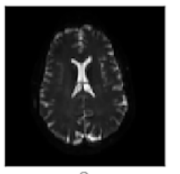

0

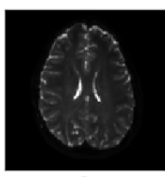

0

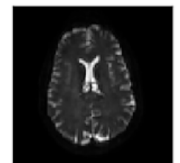

100

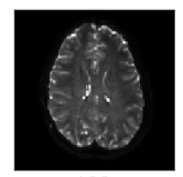

100
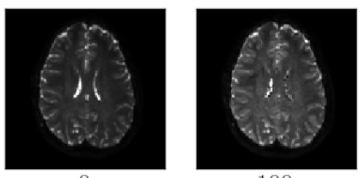

100

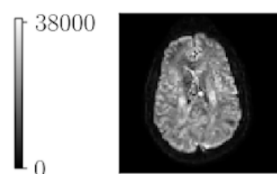

700

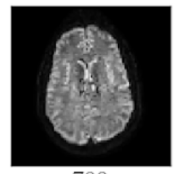

700

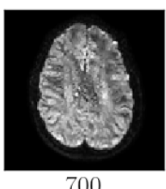

700

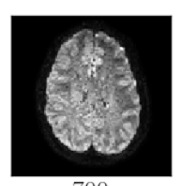

700

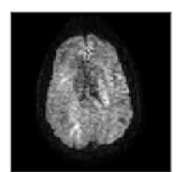

1200

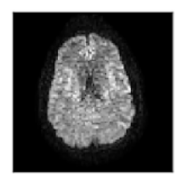

1200

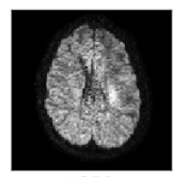

1200

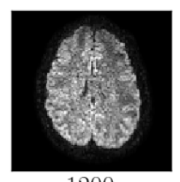

1200
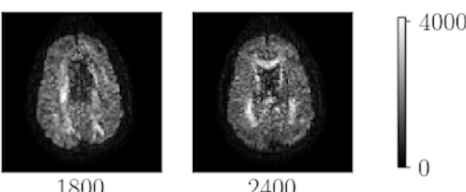

1800

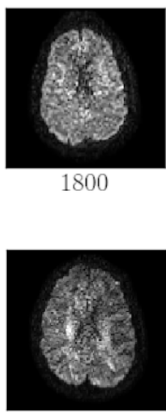

1800

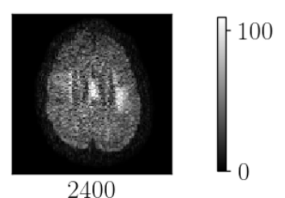

2400

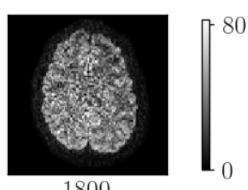

1800

Figure A.15: Examples of the raw data used in this study. The two first rows are images from the 2.5 $\mathrm{mm}$ isotropic dataset, with the first of these row being the $\mathrm{L}$ gradient table and the second one being $\mathrm{S}_{2}$. The two last rows are images from the $2.0 \mathrm{~mm}$ isotropic dataset, with the first of these row being the $\mathrm{L}$ gradient table and the second one being $\mathrm{S}_{2}$. Under each image is the b-value, in $\mathrm{s} / \mathrm{mm}^{2}$. 